This document is confidential and is proprietary to the American Chemical Society and its authors. Do not copy or disclose without written permission. If you have received this item in error, notify the sender and delete all copies.

\title{
Multivalent glycosylation of fluorescent gold nanoclusters promotes increased human dendritic cell targeting via multiple endocytic pathways
}

\begin{tabular}{|r|l|}
\hline Journal: & ACS Applied Materials \& Interfaces \\
\hline Manuscript ID & am-2015-06541y.R1 \\
\hline Manuscript Type: & Article \\
\hline Date Submitted by the Author: & $28-$ Aug-2015 \\
\hline Complete List of Authors: & $\begin{array}{l}\text { Le Guével, Xavier; The Andalusian Centre for Nanomedicine and } \\
\text { Biotechnology (BIONAND), Therapeutic Nanosystems } \\
\text { Perrez Perrino, Monica; University of Seville, Glycosystems Laboratory, } \\
\text { Instituto de Investigaciones Químicas (IIQ), CSIC } \\
\text { Fernández, Tahia; Málaga Regional University Hospital, UMA, Research } \\
\text { Laboratory and Allergy Service, IBIMA } \\
\text { Pomares, Francisca; Málaga Regional University Hospital, UMA, Research } \\
\text { Laboratory and Allergy Service, IBIMA } \\
\text { Torres, Maria Jose; Carlos Haya Hospital, } \\
\text { Blanca, Miguel; Carlos Haya Hospital, } \\
\text { Rojo, Javier; CSIC, Instituto de Investigaciones Quimicas } \\
\text { Mayorga, Cristobalina; Málaga Regional University Hospital, UMA, Research } \\
\text { Laboratory and Allergy Service, IBIMA }\end{array}$ \\
\hline \hline
\end{tabular}

\section{SCHOLARONE ${ }^{\text {'N }}$}

Manuscripts 
Multivalent Glycosylation of Fluorescent Gold Nanoclusters promotes increased Human Dendritic Cell Targeting via Multiple Endocytic Pathways

\author{
Xavier Le Guével $^{1 *}$, Monica Perrez Perrino ${ }^{2}$, Tahia D Fernández ${ }^{3}$, Francisca Pomares ${ }^{3}$, Maria- \\ José Torres ${ }^{4}$, Miguel Blanca ${ }^{4}$, Javier Rojo ${ }^{2}$, Cristobalina Mayorga ${ }^{3,4}$ \\ ${ }^{1}$ Therapeutic Nanosystems, The Andalusian Centre for Nanomedicine and Biotechnology \\ (BIONAND), Málaga, Spain \\ ${ }^{2}$ Glycosystems Laboratory, Instituto de Investigaciones Químicas (IIQ), CSIC - University of \\ Seville, Spain \\ ${ }^{3}$ Research Laboratory and Allergy Service, IBIMA, Málaga Regional University Hospital, \\ UMA, Málaga, Spain \\ ${ }^{4}$ Allergy Service, IBIMA, Regional University Málaga Hospital, UMA, Málaga, Spain \\ *Address correspondence to \\ xleguevel@bionand.es \\ Tel.: +34952367648 \\ Fax: +34952367610
}

\begin{abstract}
We report the synthesis and characterisation of gold nanoclusters (Au NCs) stabilised by a mixture of zwitterionic and multivalent mannose ligands. Characterisation of this carbohydrated nanosystem confirms its small size $(\sim 2 \mathrm{~nm})$, intense red-NIR fluorescence, relatively high affinity to lectin (ConA), and stability in physiological media. Cell studies performed using human monocyte-derived dendritic cells (DCs) show that Au NC uptake efficiency is greatly enhanced by the presence of surface carbohydrate $(>250 \%$ compared to non-carbohydrated Au
\end{abstract}


NCs) allowing their detection in cells by fluorescence following incubation with concentrations as low as $1 \mu \mathrm{g} \cdot \mathrm{mL}^{-1}$. Investigation using electron microscopy and pharmacological inhibitors indicates that $\mathrm{Au} \mathrm{NC}$ uptake is mediated by multiple endocytic pathways involving the engulfment of $\mathrm{Au}$ NCs into endosomes and partial transport to lysosomes. Results show that clathrin and F-actin dependent pathways play major roles in Au NC uptake by DCs regardless of whether or not they are coated with carbohydrates. In contrast, a specific C-lectin inhibitor induces a $60 \%$ decrease in DC particle uptake only for the carbohydrate-coated Au NCs. This study demonstrates that the combination of ultra-small gold NCs and functionalisation with multivalent mannose ligands results in greatly enhanced human DC targeting, presumably due to increased diffusion and target cell binding, respectively.

KEYWORDS: gold nanoclusters, mannose, targeting, cell uptake, dendritic cells

\section{INTRODUCTION}

As part of the development of nanoparticle (NP)-vaccines for the treatment of cancer, infectious diseases and allergies, many efforts have focused on dendritic cell (DC) targeting $^{1-3}$. DCs are one of the most potent types of antigen presenting cell (APC) and are seen as key players in the initiation and control of adaptive immune responses ${ }^{4-5}$. Furthermore, DCs reside in the periphery and in lymphoid organs, which make them ideal sentinels for pathogen recognition. As a consequence, a new generation of particulatebased vaccines are designed to i) target DCs in specific tissues, and ii) efficiently deliver antigens to DCs to either stimulate the immune response for immunotherapy or downregulate it for the treatment of allergic diseases or organ transplantation ${ }^{6-8}$. 
One of the main potential advantages of using NPs for vaccines is based on their capacity to stabilise vaccine antigens while simultaneously acting as an adjuvant ${ }^{6,9-10}$. Antigens can be encapsulated or conjugated to the NP surface, with both methods offering distinct advantages. For instance, the loading of allergens inside NPs is an elegant strategy to protect the therapeutic agent from clearance at the injection site ${ }^{11}$, while surface conjugation enhances immune cell antigen presentation ${ }^{12}$. In addition, NPs are attractive for clinical and biological applications due to their low immunogenicity, low toxicity, and good biocompatibility ${ }^{9}$. In other words, NPs have the potential, at least in theory, to deliver antigens efficiently to DCs by different pathways due to our ability to functionalise their surface with biomolecules and their capacity to modulate the immune response. Extensive research has focused on a plethora of biodegradable (PLGA, dendrimers, chitosan, virus-like-particles, liposomes) ${ }^{1,13-15}$ and non-degradable (gold, silicon, polystyrene $)^{16-18}$ NPs designed to reach DCs in in vitro and in vivo models. Two major NP parameters are considered crucial for efficient DC targeting: 1) NP size, and 2) NP surface functionalisation. Size has been shown to drastically influence particle recognition by the immune system where particles bigger than $1 \mu \mathrm{m}$ are rapidly phagocytised by macrophages ${ }^{6}$. Studies performed in in vivo models have demonstrated that NPs enter lymphatic capillaries and are taken up by peripheral DCs close to the injection site with higher uptake efficiency for particles in the range of 20-200 $\mathrm{nm}^{11,19}$. Therefore, smaller NPs might reach a higher number of DCs due to their greater diffusion and penetration. However, determining the relative effectiveness of narrower NP size ranges has been the subject of contradictory results in in vitro and in vivo models, revealing the complexity of the different factors involved in NP uptake ${ }^{20}$. Numerous 
independent studies have shown that the physico-chemical parameters of NPs have a direct impact on passive DC targeting ${ }^{18,21}$ while the presence of functional surface groups, such as carbohydrates or antibodies, have been shown to specifically bind DC surface receptors ${ }^{22}$. This strategy has been remarkably successful, notably by targeting Clectin receptors (CLRs) using customised carbohydrate ligands. The mannose receptor (MR) and DC SIGN are the most important and best characterised CLRs present on the surface of $\mathrm{DCs}^{23}$. Carbohydrate synthesis, the DC targeting efficiency of carbohydratecoated NPs and their biological applications have been covered by several excellent reviews $^{2,24}$. One elegant approach to enhance CLR targeting involves taking advantage of the multivalency of carbohydrate compounds. For example, Rojo et al. designed different glycosystems based on the attachment of multivalent mannoses or fucoses to nanocarriers (dendrimers ${ }^{25}$, fullerenes ${ }^{26}$ ) and demonstrated their high uptake efficiency by targeting DC-SIGN receptors. They also showed how these multivalent "glyconanosystems" could be applied to inhibit viral infections such as $\mathrm{HIV}^{27}$ and the Ebola virus with very high efficiency and selectivity ${ }^{26,28-29}$.

Metal (Au, Ag, Pt, Cu) nanoclusters (NCs) are a family of particles composed from just a handful of atoms up to $\sim 100$ atoms, thus spanning the gap between individual atom and nanoparticle properties. NCs exhibit unique optoelectronic properties due to their strong quantum confinement in this size regime $(\sim 0.5 \mathrm{~nm} \text { for } \mathrm{Au} \text { and } \mathrm{Ag})^{30-31}$. Recently, numerous $\mathrm{Au}$ and $\mathrm{Ag} \mathrm{NCs}$ have been reported that exhibit intense luminescence in a broad spectral range stretching from UV to near infrared (NIR) ${ }^{31}$. Such nanostructures require stabilising agents, or templates, to maintain their stability in solution and prevent the loss of their unique optical properties. Commonly reported templates for $\mathrm{Au}$ and $\mathrm{Ag}$ 
NCs include dendrimers ${ }^{32}$, biomolecules (DNA, proteins, peptides, etc.) ${ }^{33}$ and thiolated molecules ${ }^{34}$. In the latter example, extensive experimental studies supported by theoretical models ${ }^{30,35}$ demonstrate that both the NC core and the thiolated gold (I) shell contribute to their photoluminescence properties, with the shell being especially important for red-NIR region emissions ${ }^{36-39}$. For instance, the Mattoussi lab reported the preparation of red-emitting $\mathrm{Au}$ NCs with a quantum yield of up to $14 \%$ when stabilised with bidentate thiolated ligands containing a zwitterionic group ${ }^{40}$. NC features such as tunable photoluminescence ${ }^{41-42}$, multiexponential fluorescence lifetime ${ }^{43-44}$, or aggregation induced enhancement ${ }^{45}$ have been extensively studied with potential applications in the fields of sensing ${ }^{46}$, bioimaging ${ }^{47}$ and optics ${ }^{48}$. Other interesting properties of NCs relevant for therapeutic applications rely on their ultra-small size, low toxicity and stability in physiological media. In vitro studies in mice have demonstrated the rapid and efficient clearance of glutathione stabilised-Au NCs due to their size being smaller than the kidney filtration threshold $(\varnothing \sim 5.5 \mathrm{~nm})^{49-50}$. NCs have shown also promising results for cancer therapy $^{49,51}$ and vaccine development ${ }^{52}$. For example, we have recently shown how their small size and the use of a zwitterionic ligand promotes their uptake by monocyte-derived DCs and induces a strong immunosuppressive effect ${ }^{52}$, which we believe to be related to the dual action of the high diffusion of the particle in solution and the ligand contribution.

In the present study we decided to combine the ultra-small size of Au NCs $(\sim 2 \mathrm{~nm})$ and the multivalency of a mannose ligand in the presence of monocyte-derived DCs. Our aim was to determine the uptake efficiency of this nanosystem and study the mechanism by which $\mathrm{Au}$ NCs protected by zwitterionic or a mixture of zwitterionic and carbohydrate (i.e. trivalent mannose) ligands are uptaken (Figure 1). After comprehensively 
characterising the chemical and optical properties of multivalent mannose-stabilised Au NCs, cell studies determined a strong increase in DC uptake efficiency due to the presence of the multivalent mannose ligand. Electron microscopy data indicate that $\mathrm{Au}$ NC uptake in DCs occurs via endocytic pathways with particle accumulation visible in endosomes and lysosomes. Results from experiments using pharmacological inhibitors of different cellular pathways confirm the endocytic uptake of Au NCs by multiple pathways with a strong contribution from clathrin and F-actin dependent mechanisms. The specific uptake of multivalent mannose stabilised Au NCs by C-lectin receptors was also clearly demonstrated by a $60 \%$ decrease in the presence of mannan, a specific carbohydrate inhibitor.

\title{
MATERIALS AND METHODS
}

\begin{abstract}
All chemical products were purchased from Sigma Aldrich (Spain). Ultra-pure MilliQ water was used for all syntheses.
\end{abstract}

Synthesis of the ligands. Thioctic-zwitterion ( $\mathrm{Zw}, \mathrm{M} \sim 412$ g.mol-1) was synthesized following the protocol described elsewhere ${ }^{40,53}$. Synthesis of the dithiol trivalent mannose linker ( $\mathrm{M} \sim 2377$ g. $\mathrm{mol}^{-1}$ ) corresponding to 2 monomers, here called TriMan, (M $\sim 1190$ g. $\mathrm{mol}^{-1}$ ) is reported in the supporting information (refer to Figures S1 and S2 for details of the synthesis and characterisation by NMR, FTIR and mass spectroscopy). 
Synthesis of Au NCs. Zw ligand-stabilised Au NCs were prepared by adding gold salt $\left(\mathrm{HAuCl}_{4} \cdot 3 \mathrm{H}_{2} \mathrm{O}, 50 \mathrm{mM}\right)$ to a basic solution $(\mathrm{pH} 10)$ containing the ligand in the presence of $\mathrm{NaBH}_{4}$ reducing agent $(50 \mathrm{mM})$ and stirred for $15 \mathrm{~h}$. Zwitterion-stabilized Au NCs $(\mathrm{AuZw})$ were synthesised with a 1:10:2 Au:Zw:NaBH 4 molar ratio. The second type of NC containing the TriMan co-ligand (AuZwTriMan) was prepared using the same protocol as used for $\mathrm{AuZw}$ plus the dropwise addition of a specific amount of dithioTriMan 10 min after the addition of $\mathrm{NaBH}_{4}$. The optimum molar ratio for AuZwTriMan is $\mathrm{Au}: \mathrm{Zw}: \mathrm{NaBH}_{4}:$ TriMan= 1:10:2:0.21. Solutions were then dialysed with a $3.5 \mathrm{kDa}$ cutoff membrane (SnakeSkin, ThermoScientific) for $24 \mathrm{~h}$ to remove excess free ligands, then concentrated to $500 \mu \mathrm{g}$ gold $/ \mathrm{mL}$ with Amicon $3 \mathrm{kDa}$ cut-off filters (13,600 rpm; $20 \mathrm{~min})$ in water and kept refrigerated until use.

NC Characterization. NMR spectroscopy of AuZw, AuZwTriMan, and of the free ligands (Zw, TriMan) was performed using a Bruker Ascend ${ }^{\mathrm{TM}} 400 \mathrm{MHz}$ NMR with deuterium oxide. Freeze-dried samples were characterized by infrared spectroscopy using a Jasco FTIR-4100 from $650 \mathrm{~cm}^{-1}$ to $4000 \mathrm{~cm}^{-1}$. PAGE electrophoresis of the NCs: AuZw, AuZwTriMan on a 15\% polyacrylamide gel was carried out using the Bio-Rad mini-Protean system (Hercules, CA, USA) at $100 \mathrm{~V}$ for $120 \mathrm{~min}$. Each well was loaded with $20 \mu \mathrm{L}$ of concentrated sample mixed with $10 \mu \mathrm{L}$ of glycerol. Molecular weight was determined with a Precision Plus Protein Dual Xtra standard indicator ${ }^{\mathrm{TM}}(2-250 \mathrm{kDa})$. Presence of fragmented gold NCs and TriMan ligand mass were determined by Matrixassisted laser desorption/ionization (MALDI) on a 4700 Proteomics Analyzer Mass Spectrometer (ABSCIEX. Framingham, MA. USA). Samples were prepared with the matrix $\alpha$-Cyano-4-hydroxycinnamic acid (CHCA), $5 \mathrm{mg} / \mathrm{mL}$ in $50 \%$ acetonitrile, $0.1 \%$ 
trifluoroacetic acid (TFA) with a 1:1 volume ratio. The samples were analysed in Reflective mode and Linear middle mass positive ion mode: $20 \mathrm{kV}$ source 1 acceleration voltage. The Grid 1 voltage was set to $92.5 \%$ of the source 1 acceleration voltage. Delay time DE1 was $850 \mathrm{~ns}$, and the low mass gate was enabled with an offset of 0.0. Each data point was the sum of 20 spectra, acquired with 50 laser shots each. External calibration was carried out with a set of synthetic peptides (Sequazyme Peptide Mass Standards Kit, Calibration,Mixture3,ABSCIEX).

The metallic and organic composition of samples was determined by thermal and elemental analysis. Thermal analysis of AuZwTriMan was performed by Thermogravimetry (TG), and differential scanning calorimetry (DSC) using a METTLER TOLEDO model TGA/DSC 1 between 30 and $850^{\circ} \mathrm{C}$ at $10^{\circ} \mathrm{C} / \mathrm{min}$ with an air flux at 50 $\mathrm{mL} / \mathrm{min}$. Elemental analysis was estimated by inductively coupled plasma high resolution mass spectrometry (ICP-HRMS) on an ELEMENT XS (Thermo Fisher). Gold and sulphur concentration was determined with Thermo Element software (Thermo Fisher).

Au NC hydrodynamic diameters and zeta potentials in water, PBS buffer and RPMI 1640 $+10 \%$ Fetal Calf Serum (FCS) were analysed using a Nano ZS Zetasizer (Malvern). Absorption spectra over a 190-900 nm range were collected using a Cary 100 Bio UVvisible spectrophotometer (Varian). Steady-state fluorescence measurements were obtained with diluted samples on a Perkin Elmer LS45 Fluorescence Spectrometer. Fluorescence lifetime measurements were carried out on an Edinburgh Instruments FLS920 fluorometer equipped with an EPL-375 Edinburgh Instrument laser. Spectra were 
registered with excitation at $374.65 \mathrm{~nm}$ and emission detected at $680 \mathrm{~nm}$ (slits $=8 \mathrm{~nm})$.

Generation of Monocyte-Derived DCs. Fresh peripheral blood mononuclear cells, obtained from $40 \mathrm{~mL}$ of blood per individual, were used for monocyte purification by means of anti-CD14 microbeads following the manufacturer's protocol (Miltenyi Biotec, Germany). The CD14- cell fraction was placed in 10\% dimethyl sulfoxide and frozen for a later lymphocyte proliferation test. To generate DCs, monocytes (CD14+ cells) were incubated in complete medium (CM) containing Roswell Park Memorial Institute 1640 medium (Life Technologies, USA) supplemented with 10\% Fetal Calf Serum (FCS; Life Technologies, USA), streptomycin $\left(100 \mu \mathrm{g} \cdot \mathrm{mL}^{-1}\right)$, gentamicin $\left(1.25 \mathrm{U}_{\mathrm{mL}}{ }^{-1}\right)$ as well as recombinant human rhGM-CSF (200 ng. $\left.\mathrm{mL}^{-1}\right)$ and rhIL-4 (100 ng.mL $\left.{ }^{-1}\right)$ (both from R\&D Systems Inc., USA) for 5 days at $37^{\circ} \mathrm{C}$ and $5 \% \mathrm{CO} 2$. The resulting DCs were then recovered and used in the experiments.

Gold concentration in DCs. Quantification of Au NCs in DCs was estimated by inductively coupled plasma high resolution mass spectrometry (ICP-HRMS) on an ELEMENT XS (Thermo Fisher) after digesting cells (average of 70,000 cells per sample) with strong acid. Gold concentration in $\mu \mathrm{g} / \mathrm{L}$ was determined with Thermo Element software (Thermo Fisher).

Flow cytometry-based detection of Au NC-containing DCs. DCs were incubated at $1 \times 10^{5}$ cells/well in 96-well plates (Nunc, Roskilde, Denmark) with Au NCs at 1, 5, 10 and 
$25 \mu \mathrm{g} \cdot \mathrm{mL}^{-1}$ concentrations in $\mathrm{CM}$ for $3 \mathrm{~h}$ and $48 \mathrm{~h}$ at $37^{\circ} \mathrm{C}$. Cells were then analyzed using a FACSCanto II flow cytometer (BD Biosciences, USA) selecting a detection window between 720 and $860 \mathrm{~nm}$, and the data processed using FLOWJO software (Tree Star, Inc, USA).

Cell Toxicity Analyses. The cytotoxic effects of Au NCs on DCs were determined by flow cytometry. Typically, $1 \times 10^{5}$ of DCs were incubated with NCs at $1,5,10$ and 25 $\mu \mathrm{g} \cdot \mathrm{mL}^{-1}$ in $\mathrm{CM}$ for $48 \mathrm{~h}$ at $37{ }^{\circ} \mathrm{C}$ and $5 \% \mathrm{CO} 2$. After incubation, cells were stained with Live/Dead NearIR (Life Technologies-Invitrogen, USA) for 15-20 min. Cells were then assessed by flow cytometry (FACSCanto II flow cytometer, BD Biosciences, USA) using a laser excitation wavelength of $633 \mathrm{~nm}$ to measure the distribution of fluorescence emissions. Data were analysed using FLOWJO software (Tree Star, Inc, USA). The cytotoxicity of NCs on DCs are expressed as a percentage of live cells in experimental samples versus untreated cells.

Ethical Statement. This study was approved by the institutional review board "Comisión de Ética y de Investigación del Hospital Regional Universitario de Málaga", and the experiments carried out in accordance with the Declaration of Helsinki. Oral and written informed consents for all the procedures were obtained from the subjects included in the study.

Confocal Laser Microscopy (CLSM). Following incubation with Au NCs, DCs were fixed in PBS containing 4\% paraformaldehyde for 1 hour, washed three times with PBS and stored protected from light at $4{ }^{\circ} \mathrm{C}$ until analysis. Sub-membrane actin and Nuclei (DNA) were labelled by $\sim 20$ minute incubations with $10 \mu \mathrm{M}$ Atto488-conjugated 
Phalloidin (Sigma) and $1 \mu \mathrm{g} / \mathrm{ml}$ Hoechst 33258 (Sigma), respectively. Once prepared, DCs were either mounted on glass slides in Fluoroshield mounting medium (Sigma) or transferred to optical bottom 96 well plates (Nunc) in PBS for observation by CLSM. For the lysosome staining, after fixation, cells were permeabilised with saponin $(0.1 \%$ in PBS with 2\% Bovine Serum Albumin V fraction (Sigma) followed by an overnight incubation at $4^{\circ} \mathrm{C}$ with the primary rabbit LAMP-1 (Santa Cruz Biotechnology) antibody using a 1:25 dilution followed by a $1 \mathrm{~h}$ incubation at room temperature with a secondary antirabbit Cy2-conjugated antibody (Jackson Laboratories). Cells were washed three times after each antibody incubation and finally mounted on glass slides. Samples were analysed using a Leica DM6000 inverted microscope connected to a Leica SP5 laser scanning confocal system. For the calculation of $\mathrm{Au} \mathrm{NC}$ fluorescence intensities, single optical sections were captured from unstained DCs using excitation at $488 \mathrm{~nm}$ and detection of $\mathrm{Au} \mathrm{NC}$ fluorescence emissions between 580-700 nm, with an independent brightfield channel (transmitted light) to allow cell detection. For DCs stained with phalloidin, ATTO488 and Au NC fluorescence were detected simultaneously using 488 $\mathrm{nm}$ excitation with a $\sim 495-520 \mathrm{~nm}$ detection window for ATTO488 and $\sim 600-700 \mathrm{~nm}$ for Au NCs (red shifted to avoid cross-talk from ATTO488). Hoechst 33258 was measured using $405 \mathrm{~nm}$ excitation with a $415-470 \mathrm{~nm}$ detection window (measured sequentially to avoid crosstalk in other channels).

Transmission Electron Microscopy (TEM). Au NC images were obtained on a FEI Tecnai G2 Twin TEM at $200 \mathrm{kV}$. TEM samples were prepared by placing a drop of Au NC solution onto a copper grid covered with holey carbon films. Briefly, cells were fixed in a mixture of $2 \%$ paraformaldehyde- $2.5 \%$ glutaraldehyde- $0.2 \mathrm{M}$ sucrose in $\mathrm{PB}$ at $4{ }^{\circ} \mathrm{C}$, 
overnight. After centrifugation, cell pellets were embedded in $10 \%$ gelatin, which was solidified at $4^{\circ} \mathrm{C}$ and cut into small blocks. After washing with PB, blocks were post-fixed in $1 \%$ buffered osmium tetroxide for $1 \mathrm{~h}$ at room temperature, rinsed in distilled water and dehydrated in an ethanol series, followed by embedding in low viscosity Spurr's resin (Electron Microscopy Sciences). Ultrathin sections $(70 \mathrm{~nm})$ were obtained with a Leica EM UC-7 ultramicrotome at room temperature. Electron micrographs were obtained in a Tecnai G2 20 Twin (FEI) at $100 \mathrm{kV}$, with a 4 megapixels Veleta wide-angle camera (Olympus) for general imaging, and a 16 megapixel Eagle on-axis camera (FEI) for high resolution images, using TIA software (FEI).

Cell experiment with pharmacological inhibitors. To study the mechanism involved in $\mathrm{Au} \mathrm{NC}$ uptake, we selected the following inhibitors and experimentally-determined their optimal concentrations: $5 \mu \mathrm{g} \cdot \mathrm{mL}^{-1}(10 \mu \mathrm{M})$ cytochalasin $\mathrm{D}(\mathrm{CytD}), 10 \mu \mathrm{g} \cdot \mathrm{mL}^{-1}(25 \mu \mathrm{M})$ 5-(N-ethyl-N-isopropyl)hydrochloride (EIPA), $10 \mu \mathrm{g} . \mathrm{mL}-1 \quad(33 \mu \mathrm{M})$ nocodazole, 10 $\mu \mathrm{g} \cdot \mathrm{mL}^{-1}(28.1 \mu \mathrm{M})$ chlorpromazine hydrochloride, $10 \mu \mathrm{g} \cdot \mathrm{mL}^{-1}(40 \mu \mathrm{M})$ dynasore, 100 $\mu$ g.mL $\mathrm{mL}^{-1}$ mannan. $6 \times 105 \mathrm{DCs} /$ well were first pre-treated with the different inhibitors for $30 \mathrm{~min}$ and then incubated with Au NCs at 10 and $25 \mu \mathrm{g} / \mathrm{mL}$ concentrations for $8 \mathrm{~h}$ in $\mathrm{CM}$ at $37^{\circ} \mathrm{C}$. Cells were analysed by flow cytometry and confocal microscopy using the same settings as described for the concentration experiment. Toxicity tests in the presence of the inhibitors were performed with the Live/Dead NearIR stain. Data were obtained from two independent sets of experiments each using cells from three different patients.

\section{RESULTS \& DISCUSSION}


Au NC characterisation. The NCs AuZw and AuZwTriMan were prepared according to the crystal growth/reduction method using zwitterionic thiol $(\mathrm{Zw})$ and trivalent mannose derivative (TriMan) molecules as protecting agents (Figure 1).
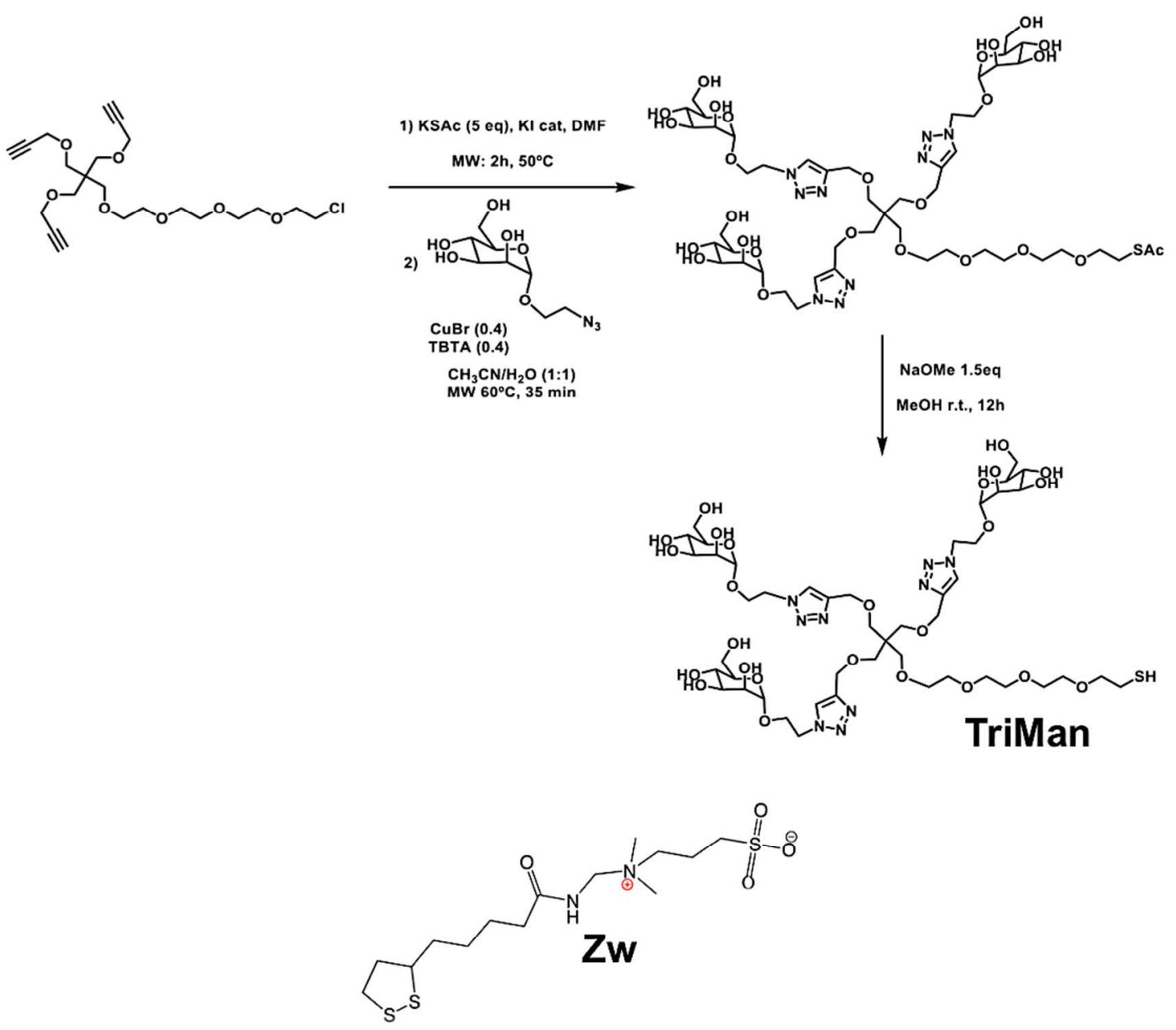

36

Figure 1. Synthetic steps for the preparation of the trivalent mannose compound (TriMan) and the chemical formula of the thioctic zwitterion compound $(\mathrm{Zw})$.

Both TriMan and Zw can interact with gold species via their mono or bidentate thiol groups, respectively. Due to the high molar Au:Ligand ratio used during synthesis, 
dialysis was performed to remove remaining free ligand and prevent self-aggregation of the thiolated ligands. Spectroscopic analyses of AuZwTriMan by NMR and FTIR (Figures S3, S4) confirmed the presence of the zwitterionic and carbohydrate molecules. Mass spectrometry (MALDI-Tof) experiments were performed on AuZw and AuZwTriMan samples using reflective and linear positive modes to obtain information on the carbohydrate ligand and the Au NCs, respectively. Our measurements in reflective mode detected the monomer signal for the trivalent mannose ligand at $\mathrm{m} / \mathrm{z}=1190.35$ with a small amount of the initial dithiol mannose compound ligand at $\mathrm{m} / \mathrm{z}=2377.66$, indicating the reduction of the dimers during synthesis (Figure S5). The measurements carried out in linear mode confirmed the presence of multiple peaks corresponding to fragmented AuZw and AuZwTriMan NCs in the same mass range, with a maximum mass of $\mathrm{m} / \mathrm{z} \sim 7000$ (Figure S6). This may indicate a similar size or structure for both NCs, where the low amount of carbohydrate added for the synthesis of AuZwTriMan does not seem to significantly influence NC growth. However, we observed a shift of the broad band centered from $\sim 14 \mathrm{kDa}$ for AuZw to $\sim 17 \mathrm{kDa}$ for AuZwTriMan. This band most likely corresponds to ligand stabilized-Au NCs with the higher molecular weight of TriMan versus $\mathrm{Zw}$ explaining the size difference. PAGE electrophoresis revealed that both fluorescent NCs migrate at $\sim 10 \mathrm{kDa}$ but with a slightly higher size distribution for AuZwTriMan (Figure S7). This observation seems to be in agreement with the mass spectrometry measurements. It should be noted that species $>10 \mathrm{kDa}$ were not visible by UV illumination, suggesting that these larger particles emit little or no fluorescence. Chemical analyses of AuZwTriMan and AuZw by thermogravimetry and elemental analysis are shown in Table 1. 


\begin{tabular}{c|cccc} 
& $\begin{array}{c}\text { Wt.\% organic } \\
\text { moiety* }\end{array}$ & Wt. \% of Man** Au:S*** & Molecular weight**** \\
\hline AuZw & $80 \pm 3$ & 0 & $1: 8.9$ & $\sim 14 \mathrm{kDa}$ \\
AuZwTriMan & $83 \pm 3$ & $8 \pm 2$ & $1: 12.5$ & $\sim 17 \mathrm{kDa}$
\end{tabular}

*: determined by thermogravimetry; **: determined by anthrone test; ***: measurements performed by ICP; ****: Value estimated for the fluorescent Au NCs by MALDI/Tof analyses.

Table 1. Chemical characterisation of the AuZwTriMan and AuZw NCs.

Our results show the high organic moiety of the sample AuZwTriMan ( $>80$ wt.\%) and an $\mathrm{Au}: \mathrm{S}$ molar ratio of $1: 12.5$, which is significantly higher than the $1: 1$ ratio suggested by the formation of organic multilayers stabilized by covalent (disulfide) and electrostatic interactions on the metal surface similar to previously reported supramolecular assemblies $^{36}$. To quantify the amount of TriMan carbohydrate in AuZwTriMan NCs, we used the colourimetric anthrone test (Figure S8), which determined that trivalent mannose accounts for approximately $8 \%$ of total organic weight. Transmission electronic microscopy (TEM) images of AuZwTriMan and AuZw (Figure S9) showed only particles with diameters $<3 \mathrm{~nm}$. Dynamic light scattering (DLS) confirmed the small size of $\operatorname{AuZw}(\varnothing=1.3 \pm 0.5 \mathrm{~nm})$ and AuZwTriMan $(\varnothing=2.7 \pm 0.5 \mathrm{~nm})$ in water (Figure 2a). Zeta potential for AuZw and AuZwTriMan were similar in water and in PBS buffer (10 $\mathrm{mM} ; \mathrm{pH} 7.4$ ) with values around $-20 \mathrm{mV}$. The negative charge of the Au NCs could be attributed to the presence of sulphur trioxide from $\mathrm{Zw}$ and hydroxyl groups from TriMan. 
Lowering the $\mathrm{pH}$ to 4 , resulted in neutral AuZwTriMan species and even slightly positively charged ones in the case of AuZw, but this did not generate detectable precipitation from either NC over a period of 6 months (data not shown). This suggests that both NCs possess strong colloidal stability.

The optical features of AuZwTriMan sol are similar to AuZw (Figures 2b) with a broad fluorescence emission in the red-NIR region $\left(\lambda_{\mathrm{em}}=680 \mathrm{~nm}\right)$ and the presence of multiple excitation bands (370, 400, 450, 490, and $515 \mathrm{~nm})$ associated with intra and inter energy electronic transitions. Such broad emissions are quite common for Au NCs and are related to the presence of multiple atomic species. We found that high TriMan concentrations resulted in decreased fluorescence intensities (Figure S10). Therefore, we determined an optimal Au:Zw: $\mathrm{NaBH}_{4}$ :TriMan ratio of 1:10:2:0.21, which resulted in a relatively high level of fluorescence with an intensity loss less than $20 \%$ with respect to AuZw and colloidal stability in vitro. The effect of high TriMan concentrations could be attributed to strong interactions between $\mathrm{Au}^{\mathrm{III}}$ and TriMan $\mathrm{OH}$ groups and TriMan's large size relative to $\mathrm{Zw}$ causing steric hindrance that prevents the growth of fluorescent $\mathrm{Au}$ NCs. The relative amounts of two different ligands have recently been shown to influence the size and shell structure of Ag-doped-Au NCs, dramatically affecting their optical properties ${ }^{54}$. The red-shift of AuZwTriMan fluorescence could be attributed either to an altered average core size, according to the jellium model ${ }^{32}$, or to changes to the metal surface following addition of the carbohydrate ligand. Indeed, with respect to the latter possibility, several studies have shown that ligand nature can affect red-NIR region photoluminescence ${ }^{38,55}$. The excitation peaks for the two Au NCs are similarly located to those reported for other Au NCs stabilised by thiol linkers regardless of the terminal 
groups used $^{55}$. The absorbance spectra of AuZwTriMan and AuZw sols show strong UV absorption with the presence of broad and weak bands between 400 and $500 \mathrm{~nm}$ (Figure 2c). Fluorescence lifetime measurements of AuZwTriMan in water show a biexponential behaviour of $\tau_{1}=300 \mathrm{~ns}(15 \%)$ and $\tau_{2}=1.5 \mu \mathrm{s}(85 \%)$ with a microsecond range lifetime characteristic of the metal-ligand electronic transition (Figure 2d) ${ }^{44,56}$. The short nanosecond range lifetime is usually associated with singlet excited states originating from metal-metal interactions. On the other hand, the long microsecond range lifetime component is typical of triplet excited states ${ }^{43,57}$. This extended high energy state is of interest for analytical applications due to its high sensitivity to the local environment. The most important optical features of AuZw and AuZwTriMan are summarised in Table 2.

a

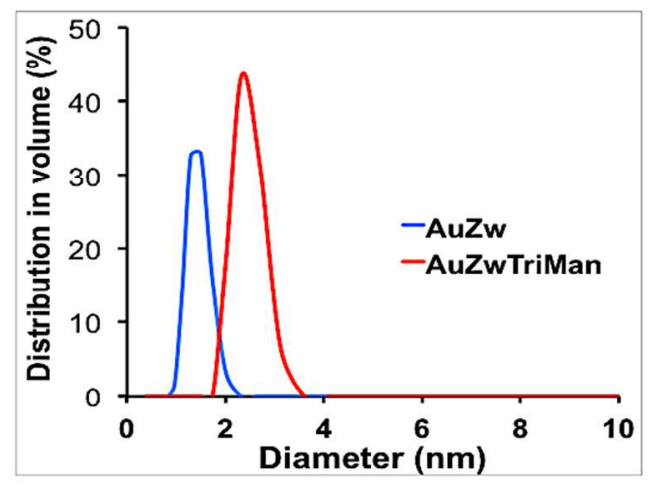

C

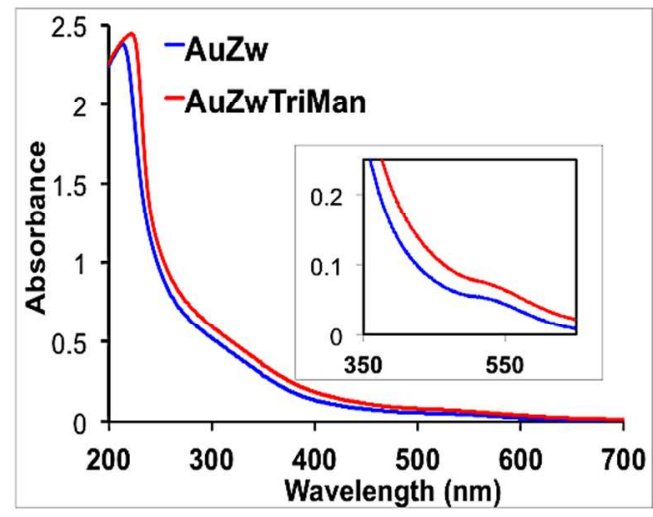

b
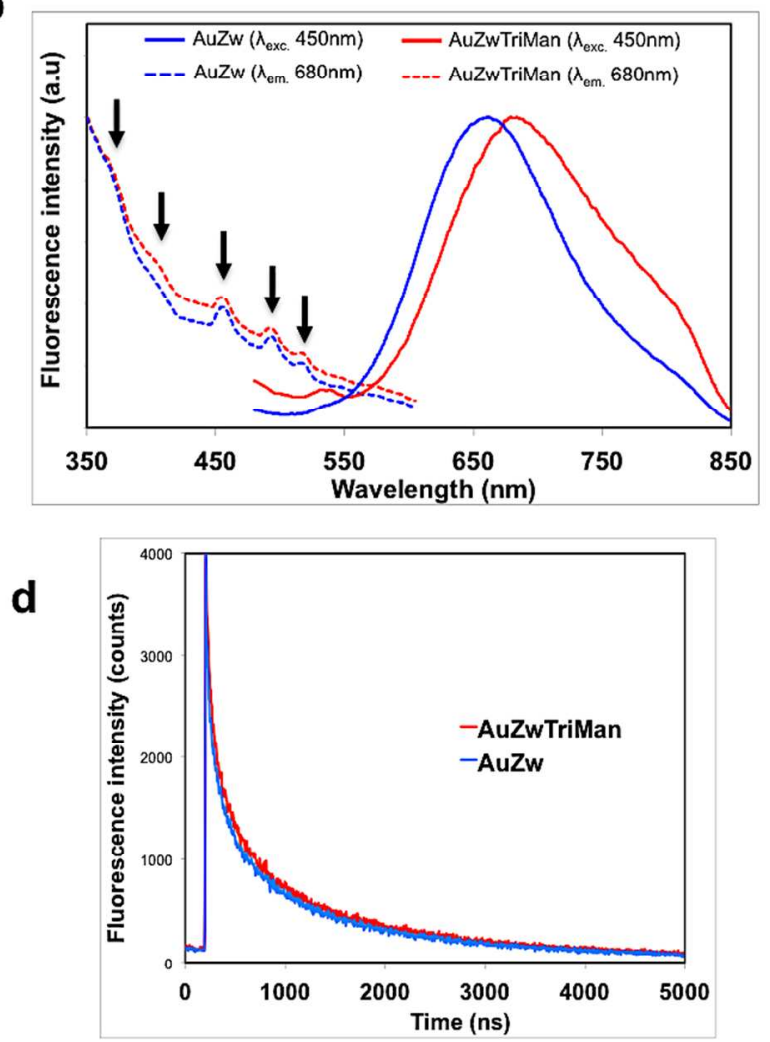

Figure 2. (a) Size measurements of AuZw and AuZwTriMan in water by dynamic light scattering (DLS). (b) Excitation (dashed line; $\lambda_{\mathrm{em}}=680 \mathrm{~nm}$ ) and emission (solid line; $\lambda_{\text {exc. }}$ $=450 \mathrm{~nm}$ ) spectra of AuZwTriMan and AuZw dispersed in water. Both Au NCs emitted in 
the red-NIR window with the typical multiple excitation peaks (370, 405, 470, $495 \mathrm{~nm}$ : arrows) of $\mathrm{Au}$ NCs. (c) The absorbance profiles of diluted AuZw and AuZwTriMan solutions exhibited strong absorbance in the UV region and a shoulder between 400 and $500 \mathrm{~nm}$ (inset). (d) Fluorescence lifetime decay of AuZwTriMan and AuZw sols. $\lambda_{\text {exc./ }} \lambda_{\text {em. }}=374.8$ $\mathrm{nm} / 680 \mathrm{~nm}$.

\begin{tabular}{|c|c|c|c|c|c|c|}
\hline & \multirow[t]{2}{*}{$\lambda_{\text {em. }} *$} & \multicolumn{2}{|c|}{$\begin{array}{l}\text { Relative fluorescence } \\
\text { intensity*** }\end{array}$} & \multicolumn{2}{|c|}{ Lifetime } & \multirow[t]{2}{*}{$\phi^{* * * *}$} \\
\hline & & Water & $\begin{array}{l}\text { PBS } \\
\mathrm{M}, \mathrm{pH} 7.2)\end{array}$ & Water & $\begin{array}{c}\text { PBS } \\
(10 \mathrm{mM}, \mathrm{pH} 7.2)\end{array}$ & \\
\hline$A u Z w$ & $665 \mathrm{~nm}$ & 1 & 0.97 & $\begin{array}{l}\tau_{1}=1.53 \mu \mathrm{s}(85 \%) ; \\
\tau_{2}=312 \mathrm{~ns}(15 \%)\end{array}$ & $\begin{array}{c}\tau_{1}=1.93 \mu \mathrm{s}(73 \%) \\
\tau_{2}=529 \mathrm{~ns}(27 \%)\end{array}$ & 11.3 \\
\hline AuZwTriMan & $680 \mathrm{~nm}$ & 0.89 & 0.79 & $\begin{array}{c}\tau_{1}=1.50 \mu \mathrm{s}(85 \%) ; \\
\tau_{2}=300 \mathrm{~ns}(15 \%)\end{array}$ & $\begin{array}{l}\tau_{1}=1.90 \mu \mathrm{s}(69 \%) \\
\tau_{2}=541 \mathrm{~ns}(27 \%)\end{array}$ & 8.7 \\
\hline
\end{tabular}

$* \lambda_{\text {exc. }}=450 \mathrm{~nm}$; **: normalised to AuZw fluorescence intensity. Both NCs have an absorbance of 0.1 at $\lambda_{\text {exc. }}=450 \mathrm{~nm} ; * * *$ determined by comparison with Fluorescein $(\phi=0.79$ in $0.1 \mathrm{M} \mathrm{NaOH})$.

Table 2. Optical properties of the AuZwTriMan and AuZw NCs.

In order to verify the activity of the trivalent mannose present on the surface of the AuZwTriMan metal core, a simple test was performed by adding the lectin concanavalin A (ConA, $\left.5 \mathrm{mg} \cdot \mathrm{mL}^{-1}\right)$ to diluted sols of AuZw and AuZwTriMan in PBS buffer (10 mM; $\mathrm{pH}$ 7.4) in the presence of calcium and magnesium ions $\left(\left(\mathrm{Ca}^{2+}\right)=\left(\mathrm{Mn}^{2+}\right)=0.1 \mathrm{mM}\right)$. Only AuZwTriMan became more turbid in the presence of ConA, indicating aggregate formation (data not shown). AuZw fluorescence decreased evenly upon adding ConA (Figure 3a). In contrast, the addition of ConA increased AuZwTriMan fluorescence at $\lambda$ $=685 \mathrm{~nm}$, accompanied by a small red shift (Figure 3b). Aggregation-induced increases in fluorescence intensity at shorter wavelengths have recently been reported ${ }^{45}$. The phenomenon of aggregation induced emission (AIE) is well documented for organic luminophores ${ }^{58}$ but has only recently been reported for metal $(\mathrm{Au}, \mathrm{Cu})$ nanoclusters ${ }^{45,59}$. 
For instance, in an elegant study from the Xie laboratory it was shown that Au NC aggregation induced by the presence of ethanol or by electrostatic interactions in the presence of cadmium cations led to a drastic fluorescent enhancement ${ }^{45}$. It was proposed that the strong fluorescence of the dense aggregates was generated by both inter/intra complex aurophilic interactions and by the reduced intramolecular motion of the complex. In our case, the AIE of AuZwTriMan emissions are likely to be the result of electrostatic interactions between the lectin and the carbohydrate ligand as the NC core is strongly protected by a saturation of mono and bidentate thiol ligands.

We expected that the long fluorescence lifetime of $\mathrm{Au}$ NCs would be sensitive to their aggregation state. Indeed, their fluorescence lifetime was longer in PBS buffer than in deionized water (Table 2) probably due to Au NC crosslinking induced by the presence of salts. Moreover, we found that the microsecond range $\left(\tau_{2}\right)$ fluorescence lifetime of AuZwTriMan was strongly influenced by the presence of ConA, dropping from $1.91 \mu$ s to $1.51 \mu \mathrm{s}$, while no change was observed for AuZw (Figure 3c). This decrease could be attributed to the combined contribution of NC local polarity changes and enhanced network rigidity induced by the formation of dense aggregates. The NC crosslinking hypothesis is consistent with the altered excitation spectrum profile of AuZwTriMan in the presence of ConA (Figure 3d). In fact, the AuZwTriMan $\lambda=450 \mathrm{~nm}$ excitation peak increased significantly relative the $\mathrm{AuZw}$ equivalent, as well as the appearance of two new peaks at longer wavelength $(\lambda=545 \mathrm{~nm} ; \lambda=575 \mathrm{~nm})$. The $450 \mathrm{~nm}$ band has been mainly associated with different gold magic cluster to electronic interband transitions $(\mathrm{sp} \longleftarrow \mathrm{d})$ that are strongly involved in ligand-to-metal charge transfers (LMCTs) or ligand to metal-metal charge transfers (LMMCTs) ${ }^{55}$. Therefore, the aggregation triggered by 
ConA could be responsible for a rearrangement of the polymeric monolayer that protects the NC gold core. However, the complexity of the photoluminescence mechanisms occurring in this system via multiple intra and inter electronic transitions will require further investigation, especially by looking at the properties of monodisperse single metal NCs. In summary, the enhanced fluorescence emissions and decreased fluorescence lifetime of AuZwTriMan are consistent with its aggregation in the presence of ConA, confirming specific binding between lectin and the carbohydrate ligand.

The colloidal stability of AuZwTriMan and AuZw dispersed in CM (RPMI1640 + 10\% FCS) was determined by DLS over $48 \mathrm{~h}$. Particles rapidly increased to $\square 10 \mathrm{~nm}$, probably due to protein corona formation, but did not show any significant aggregation (Figure S11). The presence of protein in the CM influenced Au NC surface charge with their zeta potential shifting from $-20 \mathrm{mV}$ to $-10 \mathrm{mV}$. Fluorescence measurements carried out in $\mathrm{CM}$ confirmed the relatively high optical stability of both NCs (Figure S12) over $48 \mathrm{~h}$. 


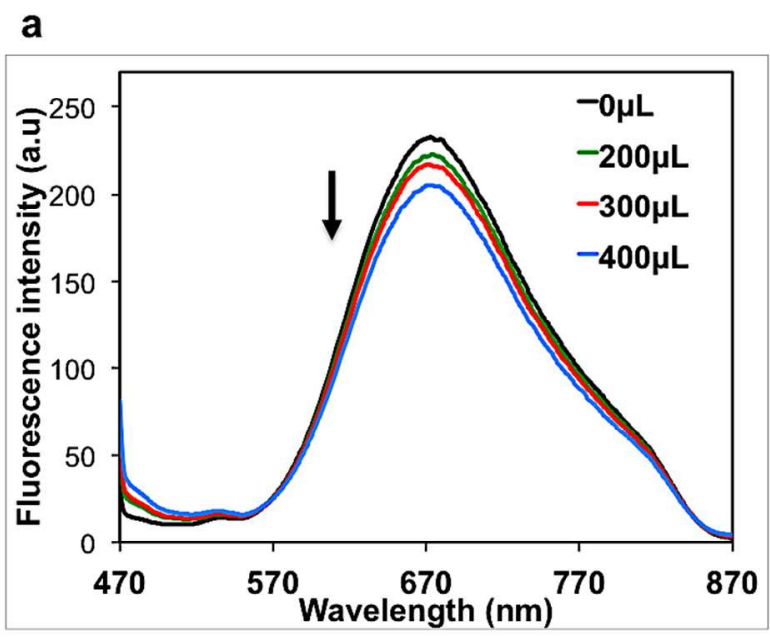

b
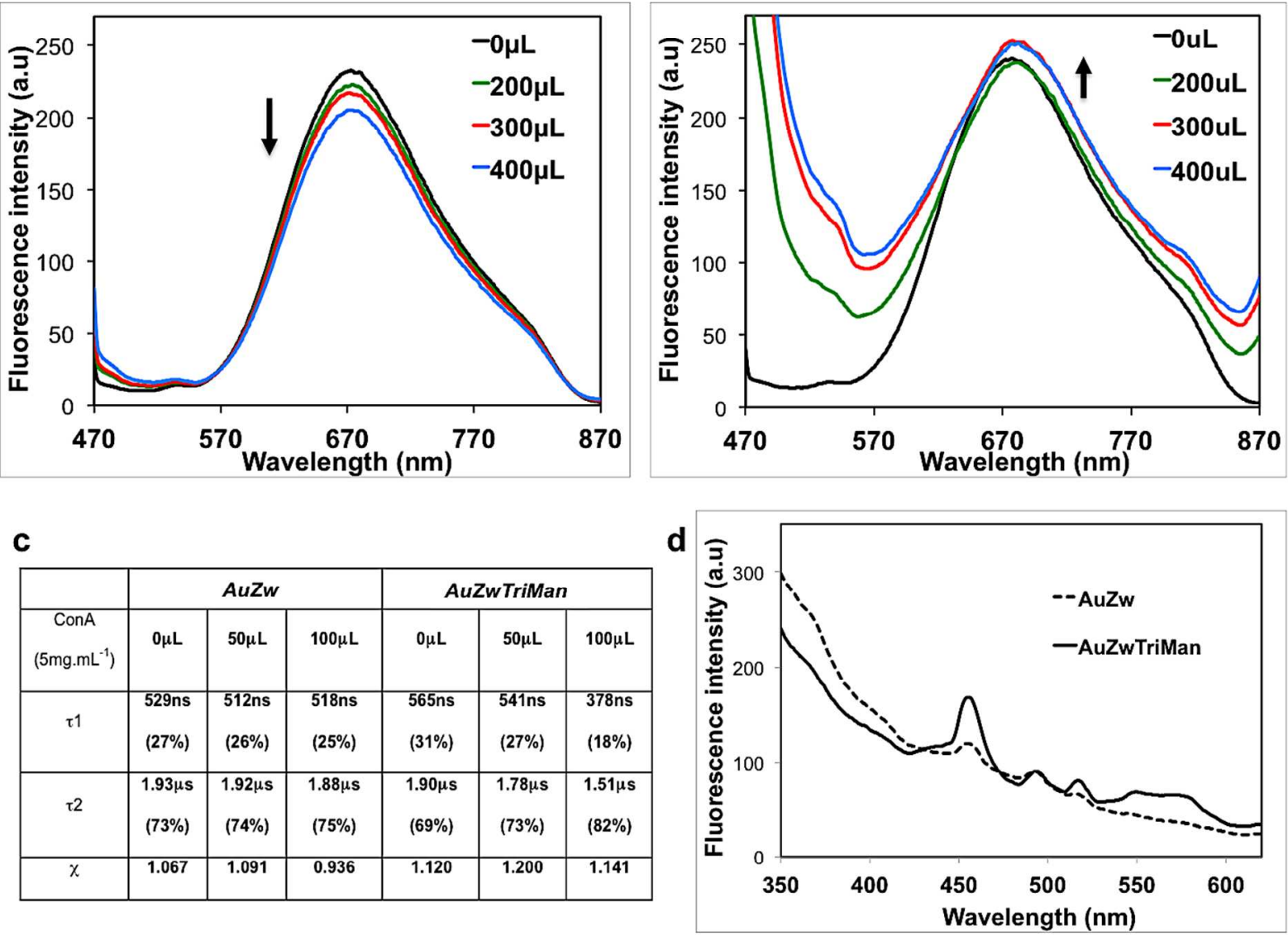

Figure 3. Fluorescence emission of AuZw (a) and AuZwTriMan (b) dispersed in PBS buffer $\left(10 \mathrm{mM}\right.$; pH 7.4) with $\left(\mathrm{Ca}^{2+}=\mathrm{Mn}^{2+}=0.1 \mathrm{mM}\right)$ after the addition of ConA (5 mg. $\mathrm{mL}^{-1}$ ). $\lambda_{\text {exc. }}=450 \mathrm{~nm}$. (c) Fluorescence lifetime data of AuZw and AuZwTriMan in the presence of ConA $\left(5 \mathrm{mg}\right.$. $\left.\mathrm{mL}^{-1}\right)$ under the same conditions as previously described. $\lambda_{\text {exc. }}=$ $374.65 \mathrm{~nm} ; \lambda_{\mathrm{em}}=680 \mathrm{~nm}$. (d) Excitation spectra of AuZw (dashed line) and AuZwTriMan (solid line) after the addition of ConA $\left(5 \mathrm{mg} \cdot \mathrm{mL}^{-1}\right) . \lambda \mathrm{em} .=680 \mathrm{~nm}$.

Au NC cellular accumulation. In order to determine the influence of the trivalent mannose linker on Au NC-DC interactions, different concentrations of AuZwTriMan and AuZw from $1 \mu \mathrm{g} \cdot \mathrm{mL}^{-1}$ to $25 \mu \mathrm{g} \cdot \mathrm{mL}^{-1}$ were incubated with DCs for $3 \mathrm{~h}$ or $48 \mathrm{~h}$ and studied by several techniques. Au NCs could be quantified and localised by flow cytometry and Confocal Laser Scanning Microscopy (CLSM) even at low concentrations thanks to their fluorescence emission in the NIR region and high Stokes-shift ${ }^{41,44}$. Flow cytometry 
measurements shown in Figure 4a indicate that AuZwTriMan and AuZw were uptaken by DCs in a dose dependent manner after $48 \mathrm{~h}$. However, the uptake of AuZwTriMan NCs was $62 \%$ (1.62 fold) stronger than for $\mathrm{AuZw}$ at $1 \mu \mathrm{g} \cdot \mathrm{mL}^{-1}$ gold concentration and by $256 \%$ (3.56 fold) at $25 \mu \mathrm{g} \cdot \mathrm{mL}^{-1}$. These results strongly suggest that the presence of TriMan promotes NC enhanced uptake in DCs. However, as fluorescence might be affected by the local cellular environment ( $\mathrm{pH}$, particle aggregation etc.), we also measured the gold concentration in DCs following incubation with AuZw and AuZwTriMan by Inductively Coupled Plasma (ICP) analysis. This analysis confirmed a higher gold concentration $(>75 \%)$ in cells incubated with the glyco-Au NC (Figure S13). These results clearly demonstrate a strong enhancement of dendritic cell NC uptake by the presence of the trivalent mannose ligand. Additional experiments found that DC uptake of both AuZwTriMan and AuZw Au NCs continues to increase after $3 \mathrm{~h}$, with higher levels of fluorescence detected after $48 \mathrm{~h}$ under most conditions (Figure S14). Interestingly, in the case of AuZwTriMan, more than $50 \%$ of cells exhibited detectable fluorescence after $3 \mathrm{~h}$ (versus only $12 \%$ for $\mathrm{AuZw}$ ) even at the lower $\mathrm{Au} \mathrm{NC}$ concentration. The CLSM images, shown in Figure 4b, indicate that NCs were distributed homogeneously in the cells and confirm the flow cytometry data with a higher uptake of AuZwTriMan than AuZw in DCs at 5 and $10 \mu \mathrm{g} \cdot \mathrm{mL}^{-1}$ concentrations after $48 \mathrm{~h}$. 


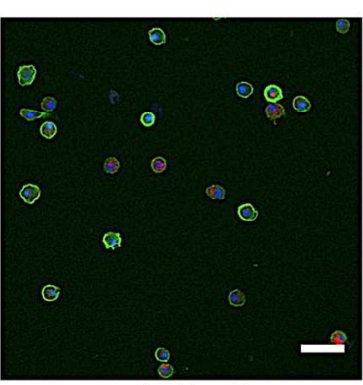

AuZw-5

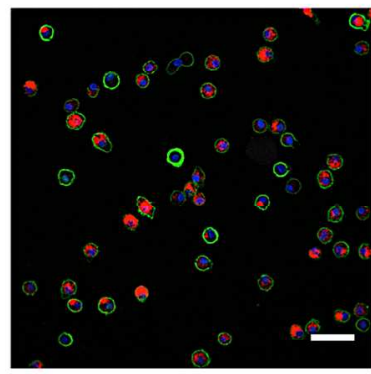

AuZwTriMan-5

a
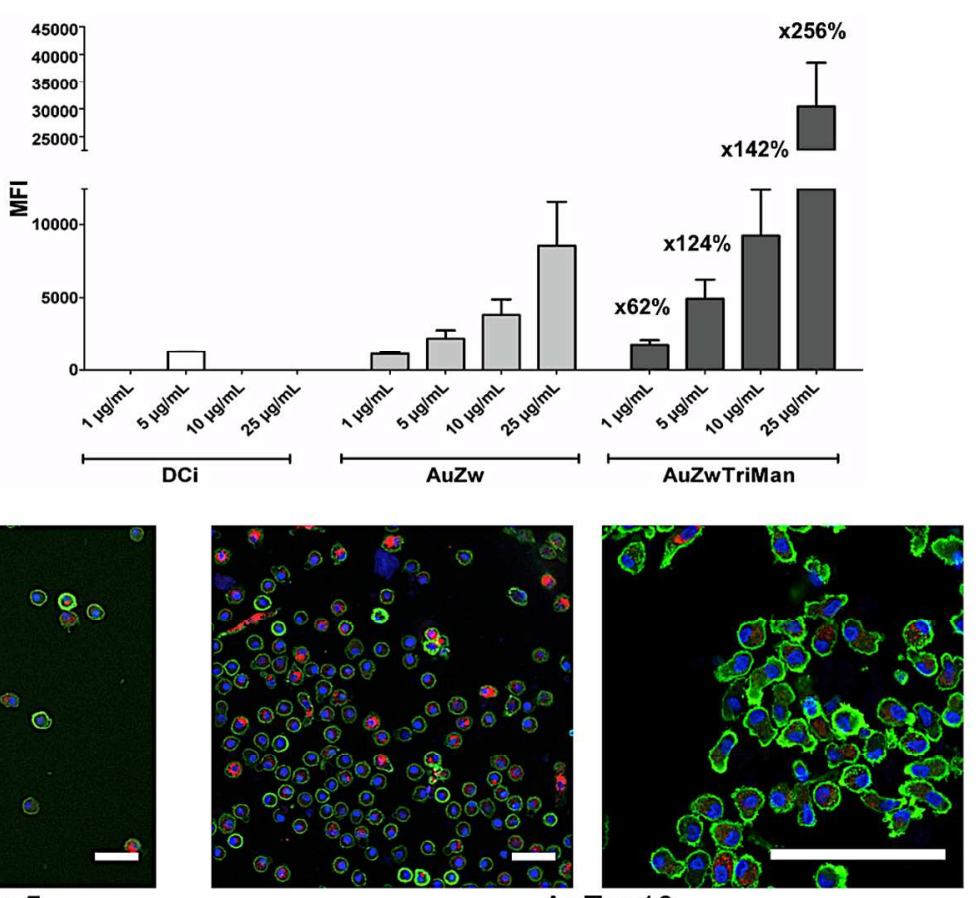

AuZw-10
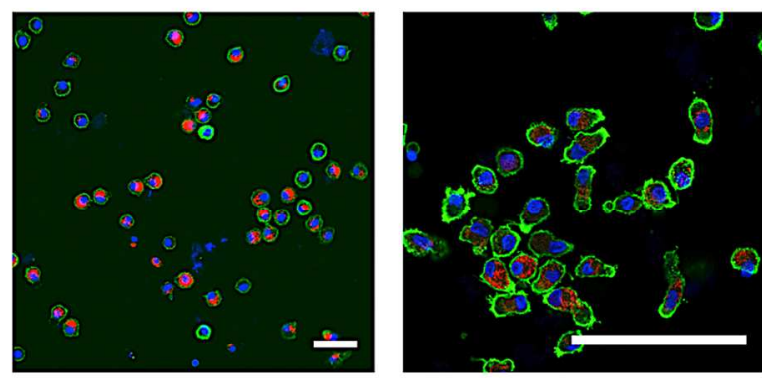

AuZwTriMan-10

Figure 4. (a) Flow cytometry measurements of DCs incubated with AuZw and AuZwTriMan at different concentrations $\left(1,5,10\right.$, and $25 \mu \mathrm{g} \cdot \mathrm{mL}^{-1}$ in CM) for $48 \mathrm{~h}$. MFI: mean fluorescence intensity. Results showed the strong enhancement of particle uptake for $\mathrm{Au}$ NCs containing the mannose ligand. (b) CLSM images of DCs incubated with AuZw and AuZwTriMan at 5 and $10 \mu \mathrm{g} \cdot \mathrm{mL}^{-1}$ in CM for $48 \mathrm{~h}$. Bars: $100 \mu \mathrm{m}$. Data are consistent with the flow cytometry analysis showing a higher fluorescence signal (red) from AuZwTriMan than for AuZw inside the cells at the same concentration. Submembrane actin was stained post-fixation with ATTO488-phalloidin (green) and nuclei with Hoechst (blue). 
The intracellular distribution of red-NIR emitting Au NCs in DCs as determined by CLSM (Figure S15) appeared similarly independent of the nature of the ligand, with both particles showing intracellular accumulation in the cytoplasm and strong perinuclear accumulation as previously reported by our $1 \mathrm{ab}^{52}$. Because both NCs contain a high content of zwitterionic ligand on their surface, the interaction between the particles and DCs will involve mainly electrostatic interactions ${ }^{53}$. TEM micrographs of DCs incubated with AuZwTriMan $\left(25 \mu \mathrm{g} \cdot \mathrm{mL}^{-1}\right.$ for $48 \mathrm{~h}$ ) show an accumulation of Au NCs (dark dots about $\square 2 \mathrm{~nm}$ in size) in different organelles (Figure $5 \mathrm{a}, \mathrm{b}, \mathrm{c}$; S16). Based on comparisons with previous particle localisation studies in DCs ${ }^{60-61}$ we identified AuZwTriMan NCs in early endosomes (a), late endosomes (a, e) and lysosomes (d, e) close to the Golgi apparatus (e), which is consistent with their uptake via endocytic pathways. Experiments with the lysosome marker LAMP-1 indicate that a relatively low proportion of Au NCs colocalise with lysosomes (Figure 5f). However, it is important to point out that only fluorescence from relatively large NC aggregates is likely to be detectable by confocal microscopy.

Cell toxicity. Next we incubated AuZw and AuZwTriMan with DCs at different concentrations $\left(1 \mu \mathrm{g} \cdot \mathrm{mL}^{-1}-25 \mu \mathrm{g} \cdot \mathrm{mL}^{-1}\right)$ and different incubation times $(3 \mathrm{~h}$ and $48 \mathrm{~h})$. Cytometry-based live/dead cell viability assays did not detect any significant toxicity above the threshold by flow cytometry measurement in any of the tested conditions (data not shown). This is consistent with absence of $\mathrm{Au}$ NC cytotoxicity described in previous studies using several in vitro and in vivo models ${ }^{49-50}$. 

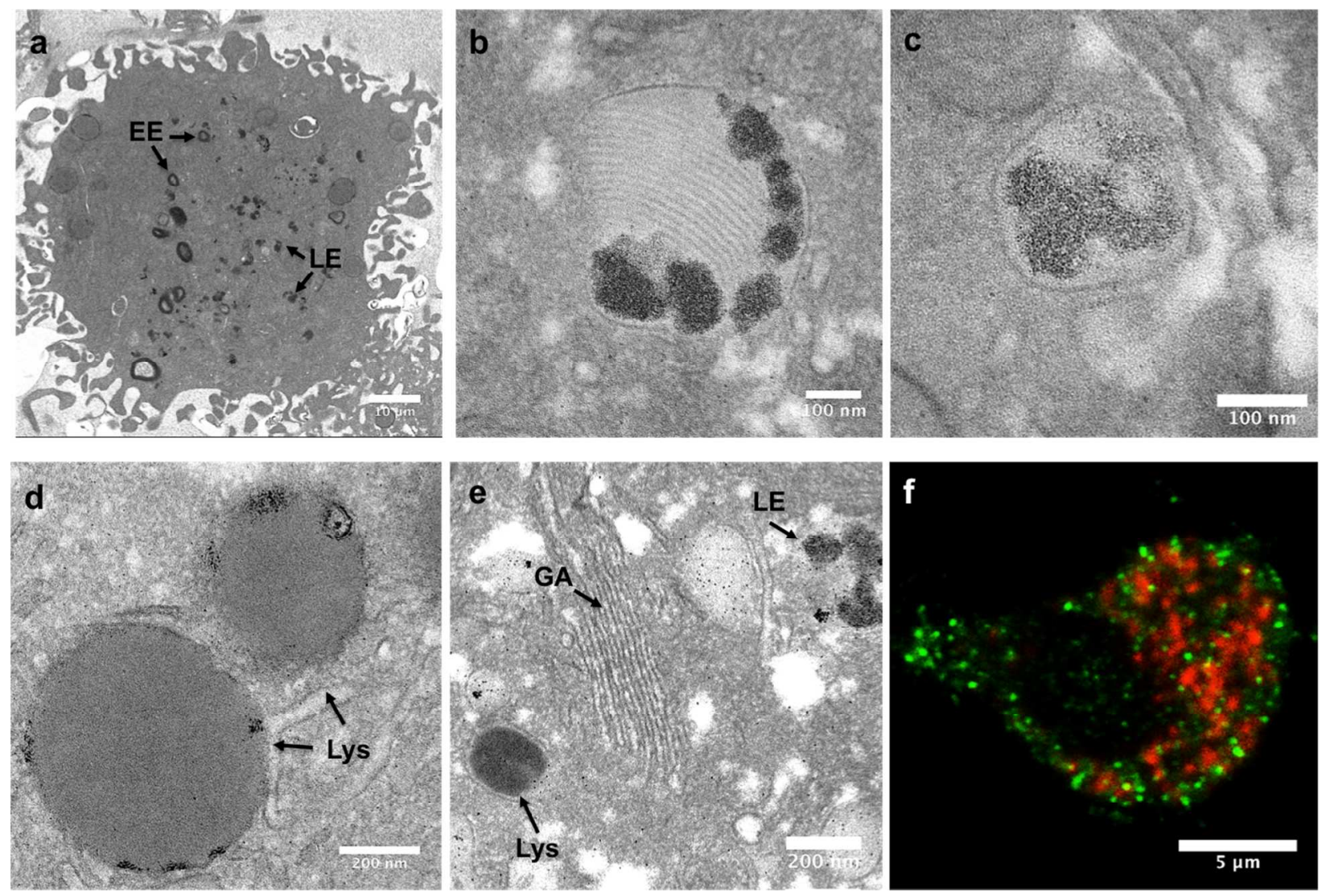

Figure 5. Electron microscopy images of DCs incubated with AuZwTriMan $\left(25 \mu \mathrm{g} \cdot \mathrm{mL}^{-1}\right.$ in CM for $48 \mathrm{~h}$ ) showing the presence of Au NCs (dark dots) in several types of multilaminar and amorphous organelles $(a, b, c)$ from early endosomes (EE) (a) and late endosomes (LE) (a, e) to lysosomes (Lys) (d, e) close to the Golgi apparatus (GA) (e). CLSM image of DCs incubated with AuZwTriMan (red), (f) stained with anti-LAMP-1 (lysosome marker, green) indicating that few LAMP1-positive lysosomes contain detectable levels of AuZwTriMan.

Au NC uptake mechanisms. Endocytosis refers to a conserved process whereby macromolecules or solutes are taken up by cells via the invagination of plasma membrane to form vesicles ${ }^{62}$. NPs with size $<200 \mathrm{~nm}$ are usually uptaken via endocytic pathways that can be divided into different classes: clathrin-dependent, caveolae, macropinocytosis, and clathrin- and caveolae-independent endocytosis ${ }^{63}$. After endocytosis, NPs tend to be 
contained within different types of intracellular vesicles, some of which can subsequently fuse with lysosomes where they accumulate until they are eventually degraded ${ }^{63}$. It has been established that cellular NP uptake mechanisms are dependent on the physicochemical parameters (size, shape, charge, hydrophobicity) of NPs, and on the cell type studied. To gain more insight into the mechanisms involved in DC uptake of Au NCs stabilised by the Zw and TriMan ligands, we selected a series of different inhibitors that differentially affect the endocytic pathways mentioned above. To optimise inhibitor concentration we first tested different levels of each inhibitor with DCs, with and without $\mathrm{Au}$ NPs, and selected the highest concentration that did not induce detectable cytotoxicity (Figure S17; data not shown). CLSM was also used to ensure an intact cell cytoskeleton (except for actin and tubulin depolymerisation using CytD and nocodazole, respectively) at different concentrations (Figure S18; data not shown). We selected an $8 \mathrm{~h} \mathrm{Au} \mathrm{NC}$ incubation time as a compromise between a short incubation time to avoid multiple uptake mechanisms and having enough fluorescent signal for cell analysis.

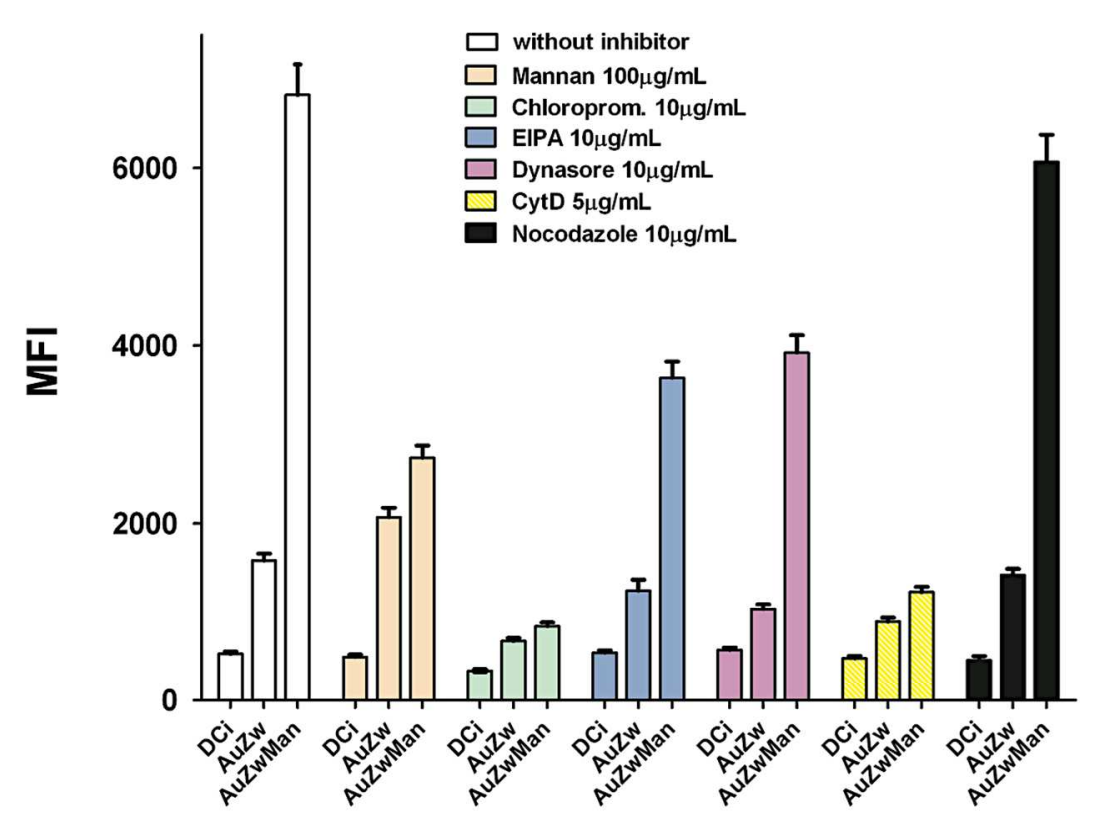


Figure 6. Endocytic pathway inhibitor experiments. DCs were pre-treated with the inhibitors for $30 \mathrm{~min}$ and then incubated for $8 \mathrm{~h}$ with either AuZw or AuZwTriMan (25 $\mu \mathrm{g} \cdot \mathrm{mL}^{-1}$ in CM). Fluorescence was measured by flow cytometry and expressed as mean fluorescence intensity (MFI).

The inhibitor experiments showed consistent results using 10 and $25 \mu \mathrm{g} \cdot \mathrm{mL}^{-1}$ of Au NCs indicating no saturation of cell uptake in this particle concentration range (Figure S19). Mannan is a compound commonly chosen as an inhibitor to confirm specific cell uptake by C-lectin receptors such as MR and DC-SIGN. Flow cytometry measurements showed a $60 \%$ reduction in $\mathrm{Au} \mathrm{NC}$ uptake for AuZwTriMan (Figure 6), while mannan had no effect on $\mathrm{AuZw}$ uptake. These data clearly demonstrate the highly specific uptake of AuZwTriMan by C-lectin receptors. This is consistent with the well-known interaction between mannose molecules and the different types of C-lectin receptor (MR, DC SIGN) present on the DC surface ${ }^{61}$. The remarkable C-lectin dependent uptake of AuZwTriMan by DCs might also be attributed to the strong affinity of DC lectin receptors for the multivalent TriMan ligand.

\begin{abstract}
A second inhibitor, chlorpromazine hydrochloride was used to determine if Au NCs are processed by clathrin-mediated endocytosis (CME). Chlorpromazine is a cationic amphiphilic class drug and an inhibitor of the clathrin-coated pit pathway. Chlorpromazine is known to cause a loss of coated pits and associated receptors from the cell surface, and result in the accumulation of clathrin and AP-2 in endosomal
\end{abstract}


compartments $^{64}$. Results showed $88 \%$ and $57 \%$ decreases for AuZwTriMan and AuZw, respectively, indicating that CME is an important pathway for their uptake by DCs. To confirm this, we tested a dynamin-inhibitor, dynasore, as dynamin is essential for clathrin-coated vesicle formation ${ }^{65}$. Dynasore tends to interfere with the GTPase activity of Dynamin and suppresses the pinching-off process but not the formation of clathrincoated pits in contrast to chlorpromazine ${ }^{66}$. Dynasore decreased the uptake of AuZwTriMan and AuZw by $42 \%$ and $59 \%$, respectively, confirming the importance of clathrin in initiating endocytosis for both Au NCs.

EIPA, an inhibitor of macropinocytosis, reduced AuZwTriMan uptake by $47 \%$ and AuZw by $21 \%$. Macropinocytosis is a form of endocytosis that involves coordinated cytoskeletal changes at the cell surface ${ }^{67}$. This result seems to suggest a less significant contribution for macropinocytosis in $\mathrm{NC}$ processing than that reported in other studies, which have ascribed macropinocytosis a major role in the engulfment of NPs by macrophages such as $\mathrm{DCs}^{63}$. CytD is a drug that can depolymerise F-actin filaments and disrupt the cytoskeleton. CytD has been reported to interfere with macropinocytosis and caveolaedependent uptake without affecting $\mathrm{CME}^{63}$. Treatment with $\mathrm{CytD}$ induced a strong $82 \%$ decrease in AuZwTriMan uptake and a 43\% decrease for AuZw. However, results from CytD treatment need to be interpreted with caution as its action is extremely broad, affecting all endocytic pathways and as well as many other biological processes ${ }^{68}$. Our results suggest that the actin cytoskeleton plays a key role in Au NC endocytosis, which is in agreement with the results of uptake mechanism studies performed on different types of NP (QD ${ }^{69}$, polystyrene ${ }^{70}$, chitosan $\left.^{62}\right)$ with sizes smaller than $50 \mathrm{~nm}$ in DCs. Our results suggest that the endocytic uptake of AuZw by DCs occurs via multiple mechanisms with 
major roles for clathrin and F-actin dependent endocytic pathways. Similar DC uptake behaviour has been reported by Zhang and colleagues using carboxyl-coated Quantum $\operatorname{Dots}(\varnothing=18 \mathrm{~nm})^{69}$.

Microtubules are a major class of cytoskeletal fibre, responsible for vesicular trafficking among many other biological functions. The nocodazole inhibitor, which disrupts the microtubule cytoskeleton, did not strongly affect the DC uptake of either type of Au NC with an $11 \%$ decrease in both cases. This is consistent with a study performed by Dawson et al. which showed that microtubule function was required for the uptake of larger (200 $\mathrm{nm})$ but not smaller $(40 \mathrm{~nm}) \mathrm{NPs}^{70}$.

\section{CONCLUSIONS}

In summary, $\mathrm{Au}$ NCs stabilised by a mixture of zwitterionic and trivalent mannose ligands were prepared using the crystal growth/reduction method. Chemical and optical characterisation confirmed their ultra-small size $(\varnothing \sim 2 \mathrm{~nm})$, fluorescence emission in the red-NIR window and the activity of their carbohydrate ligands. Interestingly, an aggregation induced emission (AIE) enhancement was observed for Au NCs coated with the mannose ligand in the presence of the lectin ConA. Measurements of Au NCs dispersed in complete medium showed relatively high colloidal and fluorescence stability. Studies in DCs showed a greater than 2.5 fold increase in particle uptake when stabilised by a trivalent mannose ligand versus a zwitterionic ligand alone. Experiments using inhibitors that target different uptake mechanisms suggest that DCs uptake Au NCs via multiple endocytic pathways with major roles for clathrin-mediated and F-actin dependent mechanisms. The $60 \%$ reduction in the uptake of mannose-coated Au NCs by 
the presence of a C-lectin inhibitor clearly demonstrates the specific targeting of Au NCs to DCs via C-lectin receptors. Electron microscopy data supports the hypothesis that Au NCs are engulfed by endocytosis, with particles present in both endosomes and lysosomes.

Regarding future applications, carbohydrate-ligand stabilized gold nanoclusters have great potential as a delivery system due to their low cytotoxicity, good colloidal stability, and strong uptake in DCs at very low concentrations $\left(1 \mu \mathrm{g} \cdot \mathrm{mL}^{-1}\right)$ thanks to their interaction with C-lectin receptors on the DC surface and their ultra-small size (i.e. high diffusion). The original optical properties of NCs allow them to be visualised by fluorescence in the red-NIR window and potentially by other techniques such as X-ray tomography or photoacoustic imaging, as well as exploited for their photothermal properties due to their gold nature.

Supporting information. Ligand synthesis \& characterisation; additional chemical and optical characterisation of NCs, cell studies including cytotoxicity and inhibitory experiments are supplied as Supporting Information.

\section{Acknowledgements}

This work was supported by the Instituto de Salud Carlos III (ISCII) (project $\mathrm{N}^{\circ}$ CP12/03310), by the Ministerio de Economía y Competitividad (MINECO) (CTQ201123410), co-financed by European Regional Development Fund (ERDF) and by ISCIIIThematic Networks and Co-operative Research Centers: RIRAAF (RD012/0013), Junta de Andalucía (CTS-7433), and ISCIII (PI12/02481-PI/02529). Xavier Le Guevel would 
like to thank Daniel Sierra for the zwitterion ligand synthesis, Carlos Rodriguez for the mass spectrometry analysis, José Maria Montenegro for the fluorescence lifetime measurement, Juan Felix Lopez (BIONAND Nanoimaging Unit) for the TEM measurements and John Pearson (BIONAND Nanoimaging Unit) for the optical microscopy analysis and critical reading of the manuscript.

\section{REFERENCES}

1. Cruz, L. J.; Tacken, P. J.; Fokkink, R.; Joosten, B.; Stuart, M. C.; Albericio, F.; Torensma, R.; Figdor, C. G., Targeted PLGA nano- but not microparticles specifically deliver antigen to human dendritic cells via DC-SIGN in vitro. Journal of Controlled Release 2010, 144 (2), 118-126.

2. Marradi, M.; Chiodo, F.; García, I.; Penadés, S., Glyconanoparticles as multifunctional and multimodal carbohydrate systems. Chemical Society Reviews 2013, 42 (11), 47284745.

3. Novak, N., Targeting Dendritic Cells in Allergen Immunotherapy. Immunology and Allergy Clinics of North America 2006, 26 (2), 307-319.

4. Abbas, A. K.; Sharpe, A. H., Dendritic cells giveth and taketh away. Nature Immunology 2005, 6 (3), 227-228.

5. Mellman, I.; Steinman, R. M., Dendritic cells: Specialized and regulated antigen processing machines. Cell 2001, 106 (3), 255-258.

6. Hubbell, J. A.; Thomas, S. N.; Swartz, M. A., Materials engineering for immunomodulation. Nature 2009, 462 (7272), 449-460.

7. Kim, J.; Mooney, D. J., In vivo modulation of dendritic cells by engineered materials: Towards new cancer vaccines. Nano Today 2011, 6 (5), 466-477.

8. Kreutz, M.; Tacken, P. J.; Figdor, C. G., Targeting dendritic cells--why bother? Blood 2013, 121 (15), 2836-2844.

9. Dobrovolskaia, M. A.; McNeil, S. E., Immunological properties of engineered nanomaterials. Nature Nanotechnology 2007, 2 (8), 469-478.

10. Klippstein, R.; Pozo, D., Nanotechnology-based manipulation of dendritic cells for enhanced immunotherapy strategies. Nanomedicine: Nanotechnology, Biology, and Medicine 2010, 6 (4), 523-529.

11. Reddy, S. T.; Rehor, A.; Schmoekel, H. G.; Hubbell, J. A.; Swartz, M. A., In vivo targeting of dendritic cells in lymph nodes with poly(propylene sulfide) nanoparticles. Journal of Controlled Release 2006, 112 (1), 26-34.

12. Hirosue, S.; Kourtis, I. C.; van der Vlies, A. J.; Hubbell, J. A.; Swartz, M. A., Antigen delivery to dendritic cells by poly(propylene sulfide) nanoparticles with disulfide 
conjugated peptides: Cross-presentation and $\mathrm{T}$ cell activation. Vaccine 2010, 28 (50), 7897-7906.

13. García-Vallejo, J. J.; Ambrosini, M.; Overbeek, A.; van Riel, W. E.; Bloem, K.; Unger, W. W. J.; Chiodo, F.; Bolscher, J. G.; Nazmi, K.; Kalay, H.; van Kooyk, Y., Multivalent glycopeptide dendrimers for the targeted delivery of antigens to dendritic cells. Molecular Immunology 2013, 53 (4), 387-397.

14. Moffat, J. M.; Cheong, W. S.; Villadangos, J. A.; Mintern, J. D.; Netter, H. J., Hepatitis B virus-like particles access major histocompatibility class I and II antigen presentation pathways in primary dendritic cells. Vaccine 2013, 31 (18), 2310-2316.

15. Unger, W. W. J.; Van Beelen, A. J.; Bruijns, S. C.; Joshi, M.; Fehres, C. M.; Van Bloois, L.; Verstege, M. I.; Ambrosini, M.; Kalay, H.; Nazmi, K.; Bolscher, J. G.; Hooijberg, E.; De Gruijl, T. D.; Storm, G.; Van Kooyk, Y., Glycan-modified liposomes boost CD4 + and CD8 + T-cell responses by targeting DC-SIGN on dendritic cells. Journal of Controlled Release 2012, 160 (1), 88-95.

16. Chiodo, F.; Marradi, M.; Park, J.; Ram, A. F. J.; Penadés, S.; Van Die, I.; Tefsen, B., Galactofuranose-coated gold nanoparticles elicit a pro-inflammatory response in human monocyte-derived dendritic cells and are recognized by DC-SIGN. ACS Chemical Biology 2014, 9 (2), 383-389.

17. Shahbazi, M. A.; Fernández, T. D.; Mäkilä, E. M.; Le Guével, X.; Mayorga, C.; Kaasalainen, M. H.; Salonen, J. J.; Hirvonen, J. T.; Santos, H. A., Surface chemistry dependent immunostimulative potential of porous silicon nanoplatforms. Biomaterials 2014, 35 (33), 9224-9235.

18. Tomić, S.; Dokić, J.; Vasilijić, S.; Ogrinc, N.; Rudolf, R.; Pelicon, P.; Vučević, D.; Milosavljević, P.; Janković, S.; Anžel, I.; Rajković, J.; Rupnik, M. S.; Friedrich, B.; Čolić, M., Size-dependent effects of gold nanoparticles uptake on maturation and antitumor functions of human dendritic cells in vitro. PLoS ONE 2014, 9 (5).

19. Reddy, S. T.; Van Der Vlies, A. J.; Simeoni, E.; Angeli, V.; Randolph, G. J.; O'Neil, C. P.; Lee, L. K.; Swartz, M. A.; Hubbell, J. A., Exploiting lymphatic transport and complement activation in nanoparticle vaccines. Nature Biotechnology 2007, 25 (10), 1159-1164.

20. Huo, S.; Ma, H.; Huang, K.; Liu, J.; Wei, T.; Jin, S.; Zhang, J.; He, S.; Liang, X. J., Superior penetration and retention behavior of $50 \mathrm{~nm}$ gold nanoparticles in tumors. Cancer Research 2013, 73 (1), 319-330.

21. Hirsjärvi, S.; Dufort, S.; Gravier, J.; Texier, I.; Yan, Q.; Bibette, J.; Sancey, L.; Josserand, V.; Passirani, C.; Benoit, J. P.; Coll, J. L., Influence of size, surface coating and fine chemical composition on the in vitro reactivity and in vivo biodistribution of lipid nanocapsules versus lipid nanoemulsions in cancer models. Nanomedicine: Nanotechnology, Biology, and Medicine 2013, 9 (3), 375-387.

22. Moros, M.; Hernáez, B.; Garet, E.; Dias, J. T.; Sáez, B.; Grazú, V.; GonzálezFernández, A.; Alonso, C.; De La Fuente, J. M., Monosaccharides versus PEGfunctionalized NPs: Influence in the cellular uptake. ACS Nano 2012, 6 (2), 1565-1577.

23. Figdor, C. G.; Van Kooyk, Y.; Adema, G. J., C-type lectin receptors on dendritic cells and langerhans cells. Nature Reviews Immunology 2002, 2 (2), 77-84.

24. Bernardi, A.; Jiménez-Barbero, J.; Casnati, A.; De Castro, C.; Darbre, T.; Fieschi, F.; Finne, J.; Funken, H.; Jaeger, K. E.; Lahmann, M.; Lindhorst, T. K.; Marradi, M.; Messner, P.; Molinaro, A.; Murphy, P. V.; Nativi, C.; Oscarson, S.; Penadés, S.; Peri, F.; 
Pieters, R. J.; Renaudet, O.; Reymond, J. L.; Richichi, B.; Rojo, J.; Sansone, F.; Schäffer, C.; Turnbull, W. B.; Velasco-Torrijos, T.; Vidal, S.; Vincent, S.; Wennekes, T.; Zuilhof, H.; Imberty, A., Multivalent glycoconjugates as anti-pathogenic agents. Chemical Society Reviews 2013, 42 (11), 4709-4727.

25. Ribeiro-Viana, R.; García-Vallejo, J. J.; Collado, D.; Pérez-Inestrosa, E.; Bloem, K.; Van Kooyk, Y.; Rojo, J., BODIPY-labeled DC-SIGN-targeting glycodendrons efficiently internalize and route to lysosomes in human dendritic cells. Biomacromolecules 2012, 13 (10), 3209-3219.

26. Luczkowiak, J.; Muñoz, A.; Sánchez-Navarro, M.; Ribeiro-Viana, R.; Ginieis, A.; Illescas, B. M.; Martín, N.; Delgado, R.; Rojo, J., Glycofullerenes inhibit viral infection. Biomacromolecules 2013, 14 (2), 431-437.

27. Sattin, S.; Daghett, A.; Thépaut, M.; Berzi, A.; Sánchez-Navarro, M.; Tabarani, G.; Rojo, J.; Fieschi, F.; Clerici, M.; Bernardi, A., Inhibition of DC-SIGN-mediated HIV infection by a linear trimannoside mimic in a tetravalent presentation. ACS Chemical Biology 2010, 5 (3), 301-312.

28. Luczkowiak, J.; Sattin, S.; Sutkevičiute, I.; Reina, J. J.; Sánchez-Navarro, M.; Thépaut, M.; Martínez-Prats, L.; Daghetti, A.; Fieschi, F.; Delgado, R.; Bernardi, A.; Rojo, J., Pseudosaccharide functionalized dendrimers as potent inhibitors of DC-SIGN dependent ebola pseudotyped viral infection. Bioconjugate Chemistry 2011, 22 (7), 13541365.

29. Ribeiro-Viana, R.; Sánchez-Navarro, M.; Luczkowiak, J.; Koeppe, J. R.; Delgado, R.; Rojo, J.; Davis, B. G., Virus-like glycodendrinanoparticles displaying quasiequivalent nested polyvalency upon glycoprotein platforms potently block viral infection. Nature Communications 2012, 3, 1-8.

30. Jin, R., Quantum sized, thiolate-protected gold nanoclusters. Nanoscale 2010, 2 (3), 343-362.

31. Zheng, J.; Zhou, C.; Yu, M.; Liu, J., Different sized luminescent gold nanoparticles. Nanoscale 2012, 4 (14), 4073-4083.

32. Zheng, J.; Nicovich, P. R.; Dickson, R. M., Highly fluorescent noble-metal quantum dots. Annual Review of Physical Chemistry 2007, 58, 409-431.

33. Goswami, N.; Zheng, K.; Xie, J., Bio-NCs-the marriage of ultrasmall metal nanoclusters with biomolecules. Nanoscale 2014, 6 (22), 13328-13347.

34. Jiang, D. E., The expanding universe of thiolated gold nanoclusters and beyond. Nanoscale 2013, 5 (16), 7149-7160.

35. Zhu, M.; Aikens, C. M.; Hollander, F. J.; Schatz, G. C.; Jin, R., Correlating the crystal structure of A thiol-protected Au25 cluster and optical properties. Journal of the American Chemical Society 2008, 130 (18), 5883-5885.

36. Le Guével, X.; Tagit, O.; Rodríguez, C. E.; Trouillet, V.; Pernia Leal, M.; Hildebrandt, N., Ligand effect on the size, valence state and red/near infrared photoluminescence of bidentate thiol gold nanoclusters. Nanoscale 2014, 6 (14), 8091 8099.

37. Negishi, Y.; Nobusada, K.; Tsukuda, T., Glutathione-protected gold clusters revisited: Bridging the gap between gold(I)-thiolate complexes and thiolate-protected gold nanocrystals. Journal of the American Chemical Society 2005, 127 (14), 5261-5270.

38. Wang, G.; Guo, R.; Kalyuzhny, G.; Choi, J. P.; Murray, R. W., NIR luminescence intensities increase linearly with proportion of polar thiolate ligands in protecting 
monolayers of $\mathrm{Au} 38$ and $\mathrm{Au} 140$ quantum dots. Journal of Physical Chemistry B 2006, 110 (41), 20282-20289.

39. Yu, Y.; Luo, Z.; Chevrier, D. M.; Leong, D. T.; Zhang, P.; Jiang, D. E.; Xie, J., Identification of a highly luminescent Au22(SG)18 nanocluster. Journal of the American Chemical Society 2014, 136 (4), 1246-1249.

40. Aldeek, F.; Muhammed, M. A. H.; Palui, G.; Zhan, N.; Mattoussi, H., Growth of highly fluorescent polyethylene glycol- and zwitterion- functionalized gold nanoclusters. ACS Nano 2013, 7 (3), 2509-2521.

41. Le Guével, X.; Spies, C.; Daum, N.; Jung, G.; Schneider, M., Highly fluorescent silver nanoclusters stabilized by glutathione: A promising fluorescent label for bioimaging. Nano Research 2012, 5 (6), 379-387.

42. Muhammed, M. A. H.; Ramesh, S.; Sinha, S. S.; Pal, S. K.; Pradeep, T., Two Distinct Fluorescent Quantum Clusters of Gold Starting from Metallic Nanoparticles by pH-Dependent Ligand Etching. Nanoresearch 2008, 1, 333-340.

43. Le Guével, X.; Hötzer, B.; Jung, G.; Hollemeyer, K.; Trouillet, V.; Schneider, M., Formation of fluorescent metal $(\mathrm{Au}, \mathrm{Ag})$ nanoclusters capped in bovine serum albumin followed by fluorescence and spectroscopy. Journal of Physical Chemistry C 2011, 115 (22), 10955-10963.

44. Shang, L.; Azadfar, N.; Stockmar, F.; Send, W.; Trouillet, V.; Bruns, M.; Gerthsen, D.; Nienhaus, G. U., One-pot synthesis of near-infrared fluorescent gold clusters for cellular fluorescence lifetime imaging. Small 2011, 7 (18), 2614-2620.

45. Luo, Z.; Yuan, X.; Yu, Y.; Zhang, Q.; Leong, D. T.; Lee, J. Y.; Xie, J., From aggregation-induced emission of $\mathrm{Au}(\mathrm{I})$-thiolate complexes to ultrabright $\mathrm{Au}(0) @ \mathrm{Au}(\mathrm{I})$ thiolate core-shell nanoclusters. Journal of the American Chemical Society 2012, 134 (40), 16662-16670.

46. Chan, P. H.; Ghosh, B.; Lai, H. Z.; Peng, H. L.; Mong, K. K. T.; Chen, Y. C., Photoluminescent Gold Nanoclusters as Sensing Probes for Uropathogenic Escherichia coli. PLOS ONE 2013, 8 (3).

47. Wang, H. H.; Lin, C. A. J.; Lee, C. H.; Lin, Y. C.; Tseng, Y. M.; Hsieh, C. L.; Chen, C. H.; Tsai, C. H.; Hsieh, C. T.; Shen, J. L.; Chan, W. H.; Chang, W. H.; Yeh, H. I., Fluorescent gold nanoclusters as a biocompatible marker for in vitro and in vivo tracking of endothelial cells. ACS Nano 2011, 5 (6), 4337-4344.

48. Kunwar, P.; Hassinen, J.; Bautista, G.; Ras, R. H. A.; Toivonen, J., Direct laser writing of photostable fluorescent silver nanoclusters in polymer films. ACS Nano 2014, 8 (11), 11165-11171.

49. Liu, J.; Yu, M.; Zhou, C.; Yang, S.; Ning, X.; Zheng, J., Passive tumor targeting of renal-clearable luminescent gold nanoparticles: Long tumor retention and fast normal tissue clearance. Journal of the American Chemical Society 2013, 135 (13), 4978-4981.

50. Zhou, C.; Long, M.; Qin, Y.; Sun, X.; Zheng, J., Luminescent gold nanoparticles with efficient renal clearance. Angewandte Chemie - International Edition 2011, 50 (14), 3168-3172.

51. Zhang, X. D.; Chen, J.; Luo, Z.; Wu, D.; Shen, X.; Song, S. S.; Sun, Y. M.; Liu, P. X.; Zhao, J.; Huo, S.; Fan, S.; Fan, F.; Liang, X. J.; Xie, J., Enhanced tumor accumulation of Sub-2 nm gold nanoclusters for cancer radiation therapy. Advanced Healthcare Materials 2014, 3 (1), 133-141. 
52. Fernandez, T. D.; Pearson, J. R.; Pernia Leal, M.; Torres, M. J.; Blanca, M.; Mayorga, C.; Le Guével, X., Intracellular accumulation and immunological properties of fluorescent gold nanoclusters in human dendritic cells. Biomaterials 2015, 43, 1-12.

53. Park, J.; Nam, J.; Won, N.; Jin, H.; Jung, S.; Jung, S.; Cho, S. H.; Kim, S., Compact and stable quantum dots with positive, negative, or zwitterionic surface: Specific cell interactions and non-specific adsorptions by the surface charges. Advanced Functional Materials 2011, 21 (9), 1558-1566.

54. Dou, X.; Yuan, X.; Yao, Q.; Luo, Z.; Zheng, K.; Xie, J., Facile synthesis of watersoluble $\mathrm{Au}<\mathrm{inf}>25-\mathrm{x}</ \mathrm{inf}>\mathrm{Ag}<\mathrm{inf}>\mathrm{x}</$ inf $>$ nanoclusters protected by mono- and bithiolate ligands. Chemical Communications 2014, 50 (56), 7459-7462.

55. Wu, Z.; Jin, R., On the ligand's role in the fluorescence of gold nanoclusters. Nano Letters 2010, 10 (7), 2568-2573.

56. Le Guevel, X., Recent advances on the synthesis of metal quantum nanoclusters and their application for bioimaging. IEEE Journal on Selected Topics in Quantum Electronics 2014, 20 (3), 1-12.

57. Zhou, C.; Sun, C.; Yu, M.; Qin, Y.; Wang, J.; Kim, M.; Zheng, J., Luminescent gold nanoparticles with mixed valence states generated from dissociation of polymeric $\mathrm{Au}(\mathrm{I})$ thiolates. Journal of Physical Chemistry C 2010, 114 (17), 7727-7732.

58. Hong, Y.; Lam, J. W. Y.; Tang, B. Z., Aggregation-induced emission. Chemical Society Reviews 2011, 40 (11), 5361-5388.

59. Jia, X.; Li, J.; Wang, E., Cu Nanoclusters with Aggregation Induced Emission Enhancement. small 2013, 9 (22), 3873-3879.

60. Dunn, K. W.; Kamocka, M. M.; McDonald, J. H., A practical guide to evaluating colocalization in biological microscopy. American Journal of Physiology - Cell Physiology 2011, 300 (4), C723-C742.

61. Engering, A. J.; Cella, M.; Fluitsma, D.; Brockhaus, M.; Hoefsmit, E. C. M.; Lanzavecchia, A.; Pieters, J., The mannose receptor functions as a high capacity and broad specificity antigen receptor in human dendritic cells. European Journal of Immunology 1997, 27 (9), 2417-2425.

62. Nam, H. Y.; Kwon, S. M.; Chung, H.; Lee, S. Y.; Kwon, S. H.; Jeon, H.; Kim, Y.; Park, J. H.; Kim, J.; Her, S.; Oh, Y. K.; Kwon, I. C.; Kim, K.; Jeong, S. Y., Cellular uptake mechanism and intracellular fate of hydrophobically modified glycol chitosan nanoparticles. Journal of Controlled Release 2009, 135 (3), 259-267.

63. Khalil, I. A.; Kogure, K.; Akita, H.; Harashima, H., Uptake pathways and subsequent intracellular trafficking in nonviral gene delivery. Pharmacological Reviews 2006, 58 (1), 32-45.

64. Chen, C. L.; Hou, W. H.; Liu, I. H.; Hsiao, G.; Huang, S. S.; Huang, J. S., Inhibitors of clathrin-dependent endocytosis enhance TGF $\beta$ signaling and responses. $J$. Cell Sci. 2009, 122 (11), 1863-1871.

65. Macia, E.; Ehrlich, M.; Massol, R.; Boucrot, E.; Brunner, C.; Kirchhausen, T., Dynasore, a Cell-Permeable Inhibitor of Dynamin. Dev. Cell 2006, 10 (6), 839-850.

66. Shang, L.; Nienhaus, K.; Jiang, X.; Yang, L.; Landfester, K.; Mailänder, V.; Simmet, T.; Nienhaus, G., Nanoparticle interactions with live cells: Quantitative fluorescence microscopy of nanoparticle size effects. Belstein Journal of Nanotechnology 2014, 5, 2388-2397. 
67. Falcone, S.; Cocucci, E.; Podini, P.; Kirchhausen, T.; Clementi, E.; Meldolesi, J., Macropinocytosis: Regulated coordination of endocytic and exocytic membrane traffic events. J. Cell Sci. 2006, 119 (22), 4758-4769.

68. Zegers, M. M. P.; Zaal, K. J. M.; Van Ijzendoorn, S. C. D.; Klappe, K.; Hoekstra, D., Actin filaments and microtubules are involved in different membrane traffic pathways that transport sphingolipids to the apical surface of polarized HepG2 cells. Mol. Biol. Cell 1998, 9 (7), 1939-1949.

69. Zhang, L. W.; Bäumer, W.; Monteiro-Riviere, N. A., Cellular uptake mechanisms and toxicity of quantum dots in dendritic cells. Nanomedicine 2011, 6 (5), 777-791.

70. dos Santos, T.; Varela, J.; Lynch, I.; Salvati, A.; Dawson, K. A., Effects of transport inhibitors on the cellular uptake of carboxylated polystyrene nanoparticles in different cell lines. PLoS ONE 2011, 6 (9).

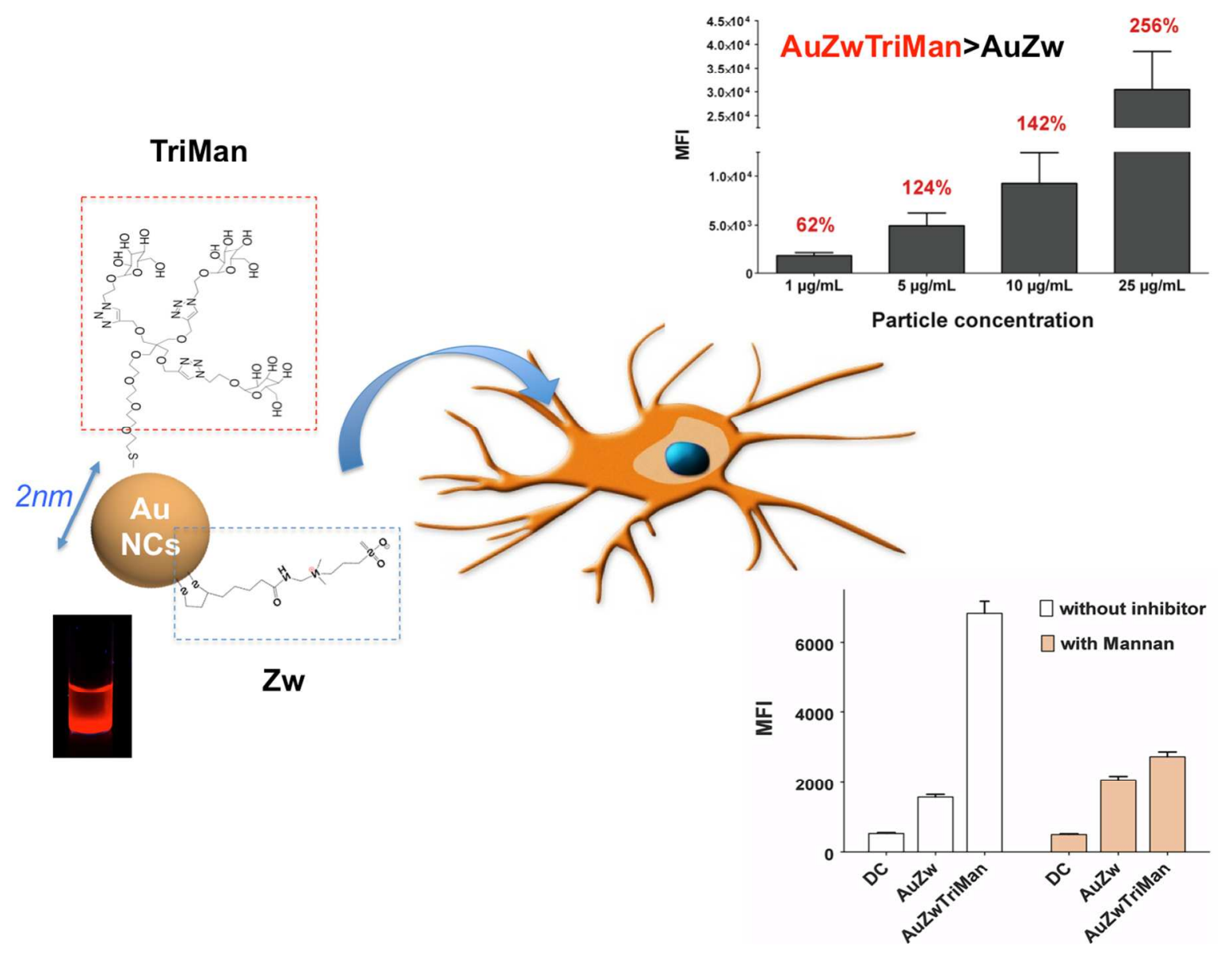

Fluorescent gold nanoclusters $($ size $\approx 2 \mathrm{~nm})$ are readily uptaken by human dendritic cells via endocytic mechanisms when stabilized by a mixture of zwitterionic and multivalent mannose ligands. This strong uptake depends specifically on interactions between the coated nanoclusters and cellular $\mathrm{C}$-lectin mannose receptors. 


\section{Page 37 of 44}

1

2

3

4

5

6

7

8

9

10

11

12

13

14

15

16

17

18

19

20

21

22

23

24

25

26

27

28

29

30

31

32

33

34

35

36

37

38

39

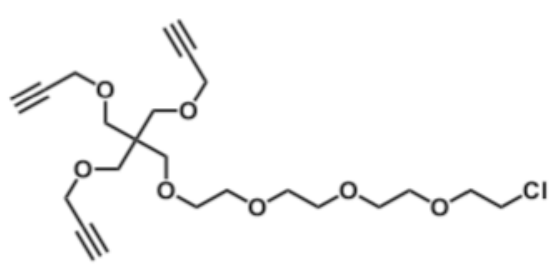

1) KSAc (5 eq), KI cat, DMF

$M W: 2 \mathrm{~h}, 50^{\circ} \mathrm{C}$

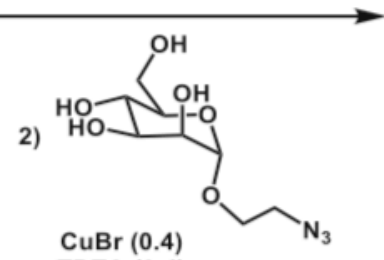

TBTA $(0.4)$

$\mathrm{CH}_{3} \mathrm{CN} / \mathrm{H}_{2} \mathrm{O}(1: 1)$

$\mathrm{MW} 60^{\circ} \mathrm{C}, 35 \mathrm{~min}$
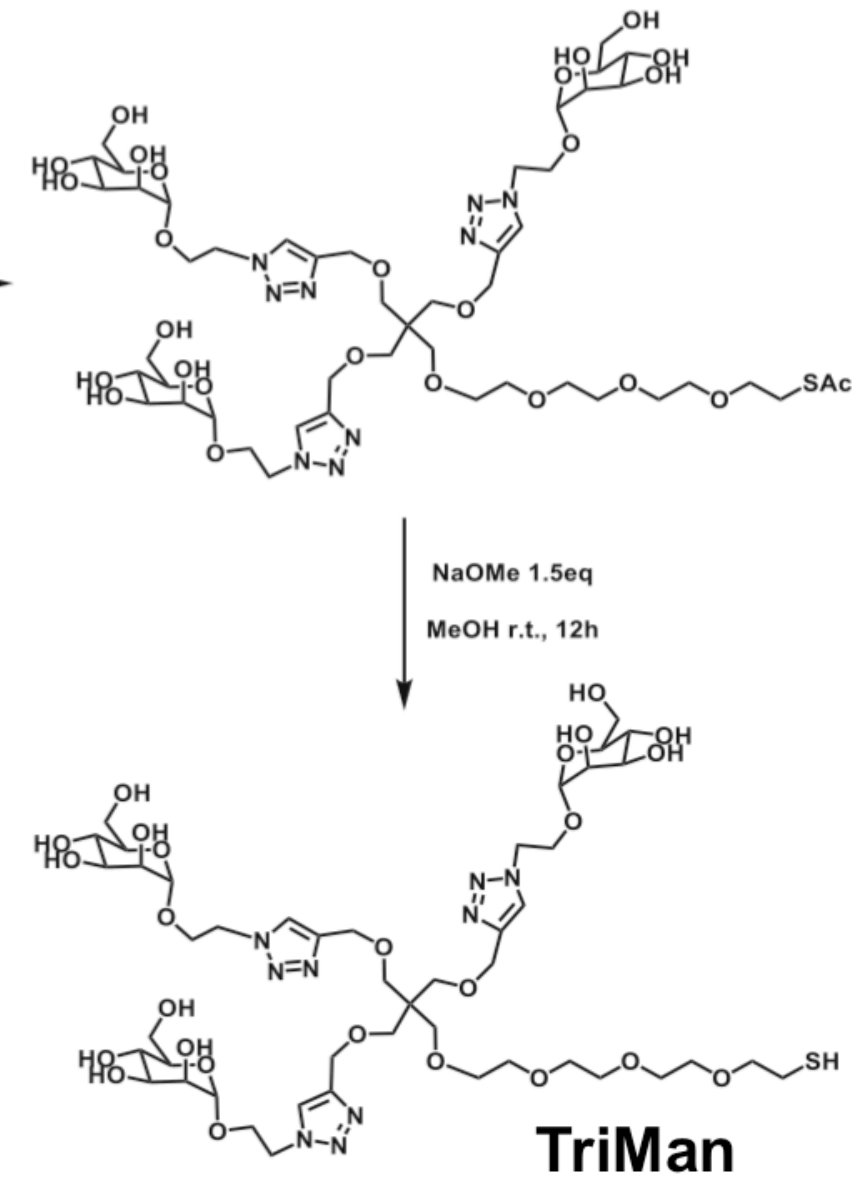

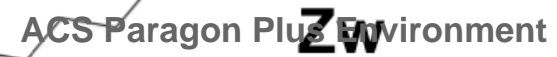




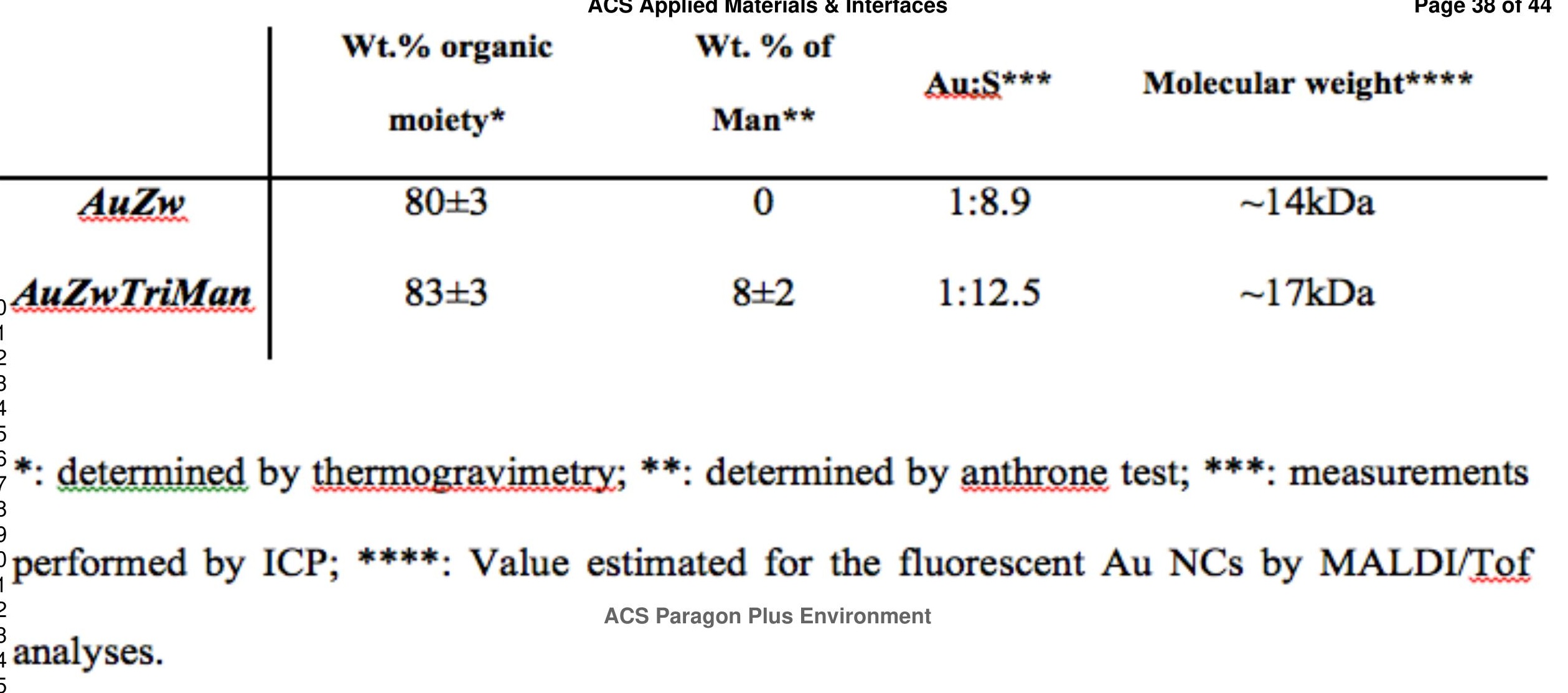




\section{Rage 39 of 44}

\section{ACS Applied M|jerials \& Interfaces}
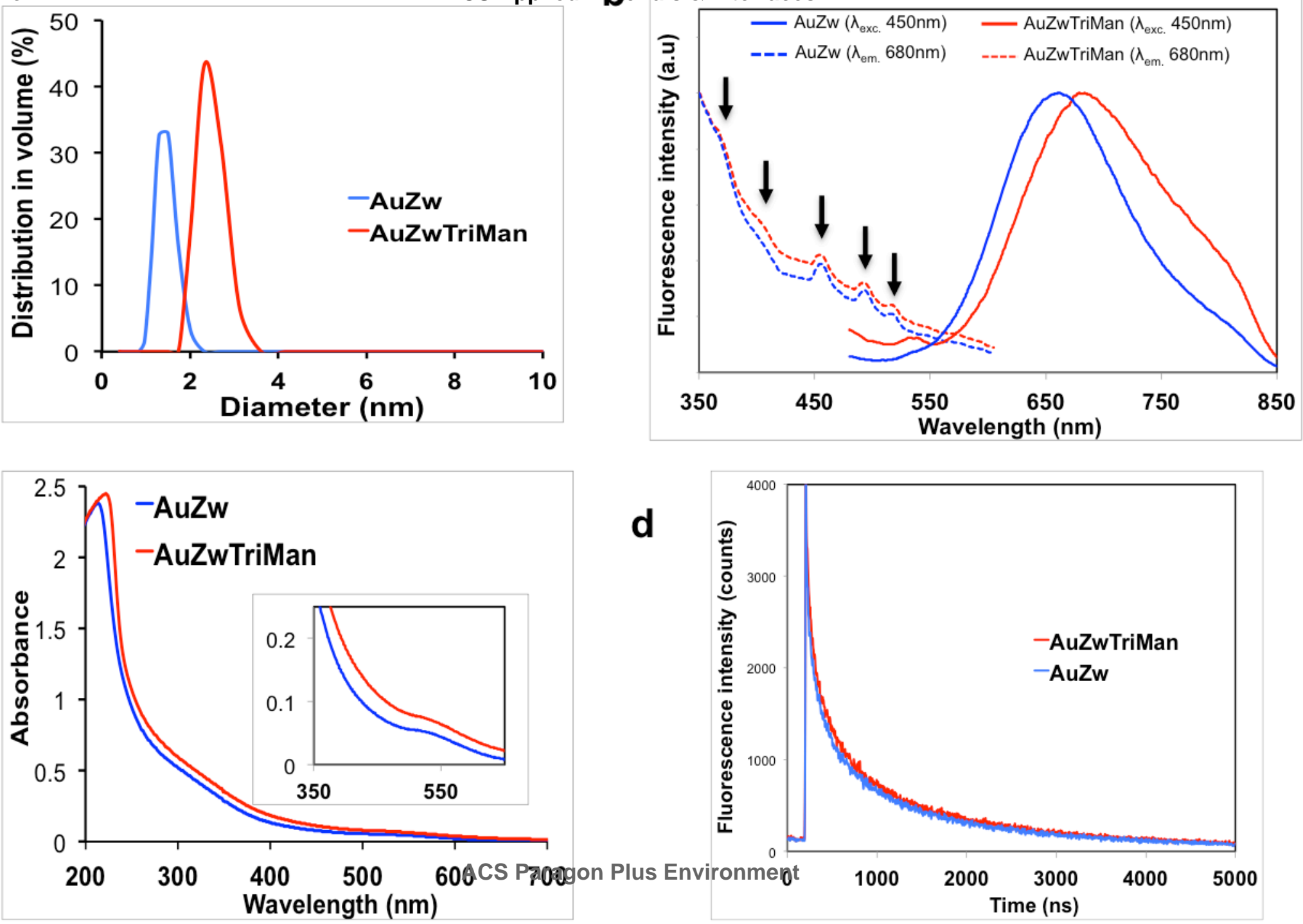
ACS Applied Materials \& Interfaces

$\lambda_{\text {oen }}$ * Relative fluorescence intensity**

\begin{tabular}{|c|c|c|c|c|c|c|}
\hline & \multirow[t]{2}{*}{$\lambda_{\text {oge }}{ }^{*}$} & \multicolumn{2}{|c|}{$\begin{array}{l}\text { Relative fluorescence } \\
\text { intensity }\end{array}$} & \multicolumn{2}{|c|}{ Lifetime } & \multirow[t]{2}{*}{$\phi^{* * *}$} \\
\hline & & Water & $\begin{array}{c}\text { PBS } \\
(10 \mathrm{mM}, \mathrm{pH} 7.2)\end{array}$ & Water & $\begin{array}{c}\text { PBS } \\
(10 \mathrm{mM}, \mathrm{pH} 7.2)\end{array}$ & \\
\hline$A u Z w$ & $665 \mathrm{~nm}$ & 1 & 0.97 & $\begin{array}{c}\tau_{1}=1.53 \mu \mathrm{s}(85 \%) ; \\
\tau_{2}=312 \mathrm{~ns}(15 \%)\end{array}$ & $\begin{array}{c}\tau_{1}=1.93 \mu \mathrm{s}(73 \%) \\
\tau_{2}=529 \mathrm{~ns}(27 \%)\end{array}$ & 11.3 \\
\hline AuZwTriMan & $680 \mathrm{~nm}$ & 0.89 & 0.79 & $\begin{array}{c}\tau_{1}=1.50 \mu \mathrm{s}(85 \%) \\
\tau_{2}=300 \mathrm{~ns}(15 \%)\end{array}$ & $\begin{array}{l}\tau_{1}=1.90 \mu \mathrm{s}(69 \%) \\
\tau_{2}=541 \mathrm{~ns}(27 \%)\end{array}$ & 8.7 \\
\hline
\end{tabular}

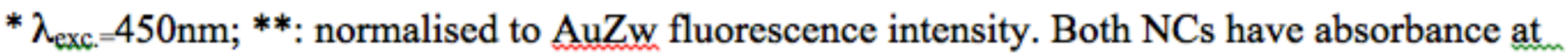
0.1 using $\lambda_{\text {exc. }}=450 \mathrm{~nm}$; *** determined by comparison with Fluorescein $(\phi=0.79$ in $0.1 \mathrm{M}$ $3 \mathrm{NaOH})$ 
ACS Applied Materiłb \& Interfaces
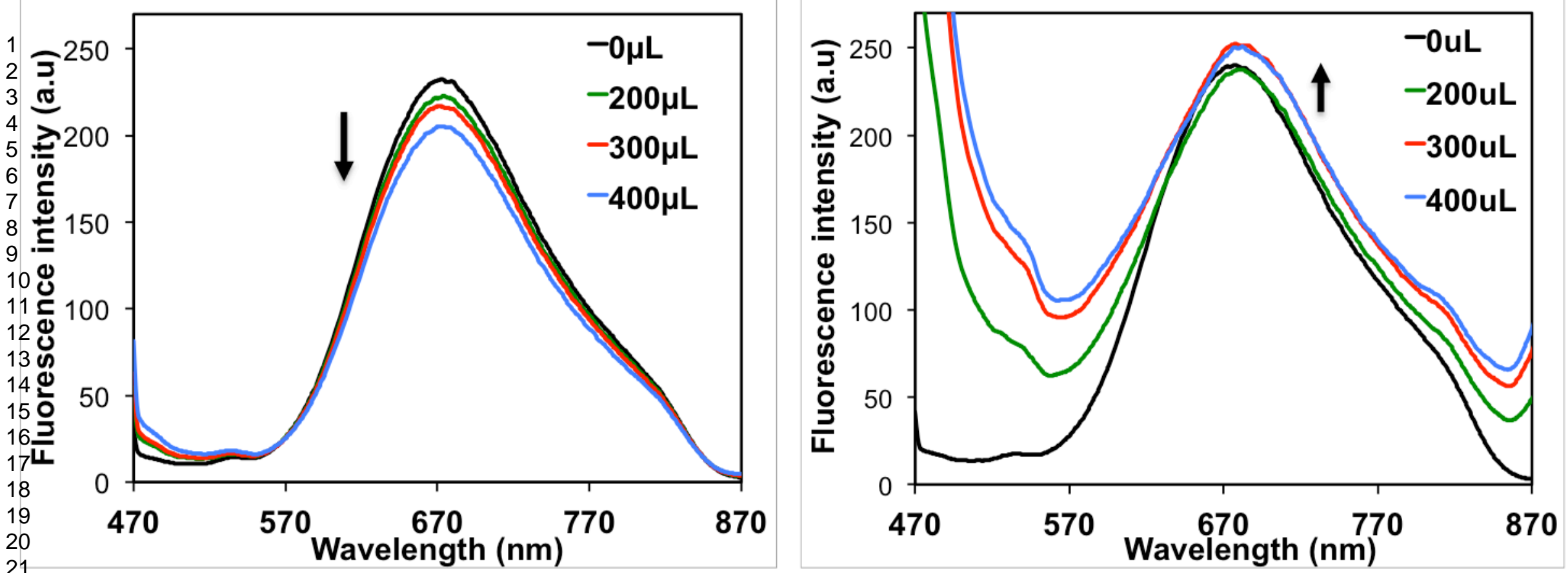

\begin{tabular}{|c|c|c|c|c|c|c|}
\hline & \multicolumn{3}{|c|}{ AuZw } & \multicolumn{3}{c|}{ AuZwTriMan } \\
\hline $\begin{array}{c}\text { ConA } \\
\left(5{\left.\mathrm{mg} \cdot \mathrm{mL}^{-1}\right)}^{-1}\right.\end{array}$ & $0 \mu \mathrm{L}$ & $50 \mu \mathrm{L}$ & $100 \mu \mathrm{L}$ & $0 \mu \mathrm{L}$ & $50 \mu \mathrm{L}$ & $100 \mu \mathrm{L}$ \\
\hline$\tau 1$ & $529 \mathrm{~ns}$ & $512 \mathrm{~ns}$ & $518 \mathrm{~ns}$ & $565 \mathrm{~ns}$ & $541 \mathrm{~ns}$ & $378 \mathrm{~ns}$ \\
& $(27 \%)$ & $(26 \%)$ & $(25 \%)$ & $(31 \%)$ & $(27 \%)$ & $(18 \%)$ \\
\hline \multirow{2}{*}{$\tau$} & $1.93 \mu \mathrm{s}$ & $1.92 \mu \mathrm{s}$ & $1.88 \mu \mathrm{s}$ & $1.90 \mu \mathrm{s}$ & $1.78 \mu \mathrm{s}$ & $1.51 \mu \mathrm{s}$ \\
& $(73 \%)$ & $(74 \%)$ & $(75 \%)$ & $(69 \%)$ & $(73 \%)$ & $(82 \%)$ \\
\hline$\chi$ & 1.067 & 1.091 & 0.936 & 1.120 & 1.200 & 1.141 \\
ACS Paragon Plus
\end{tabular}

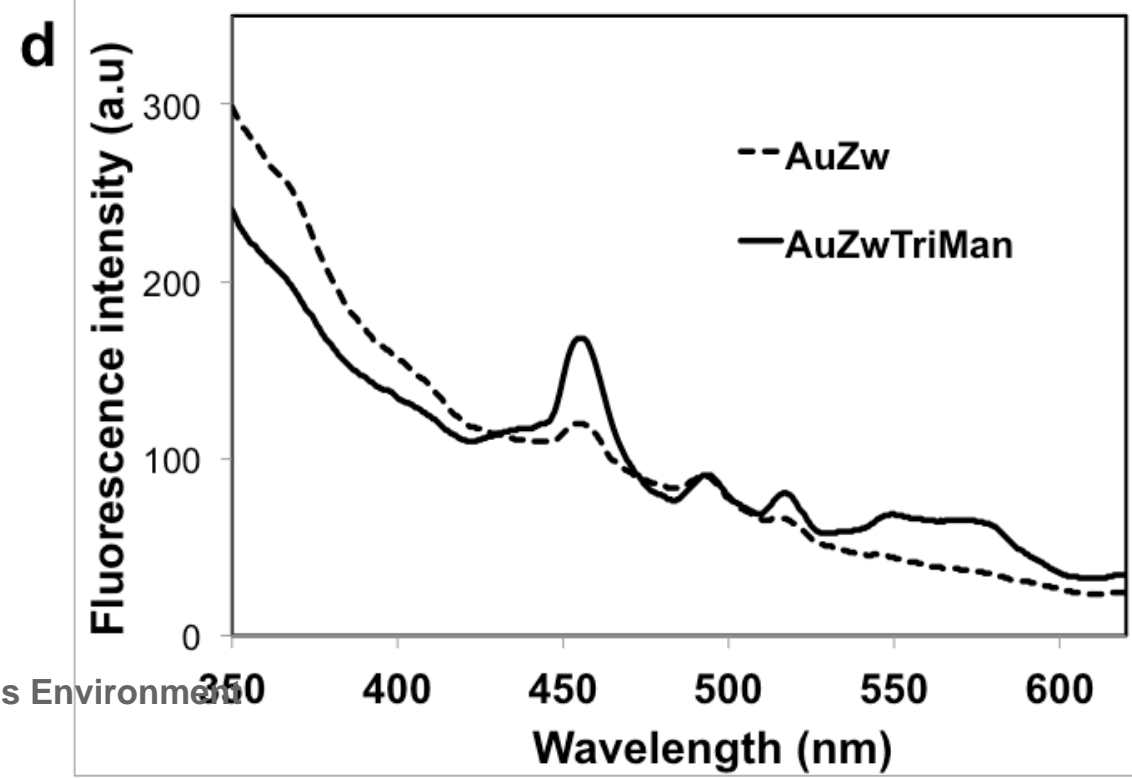



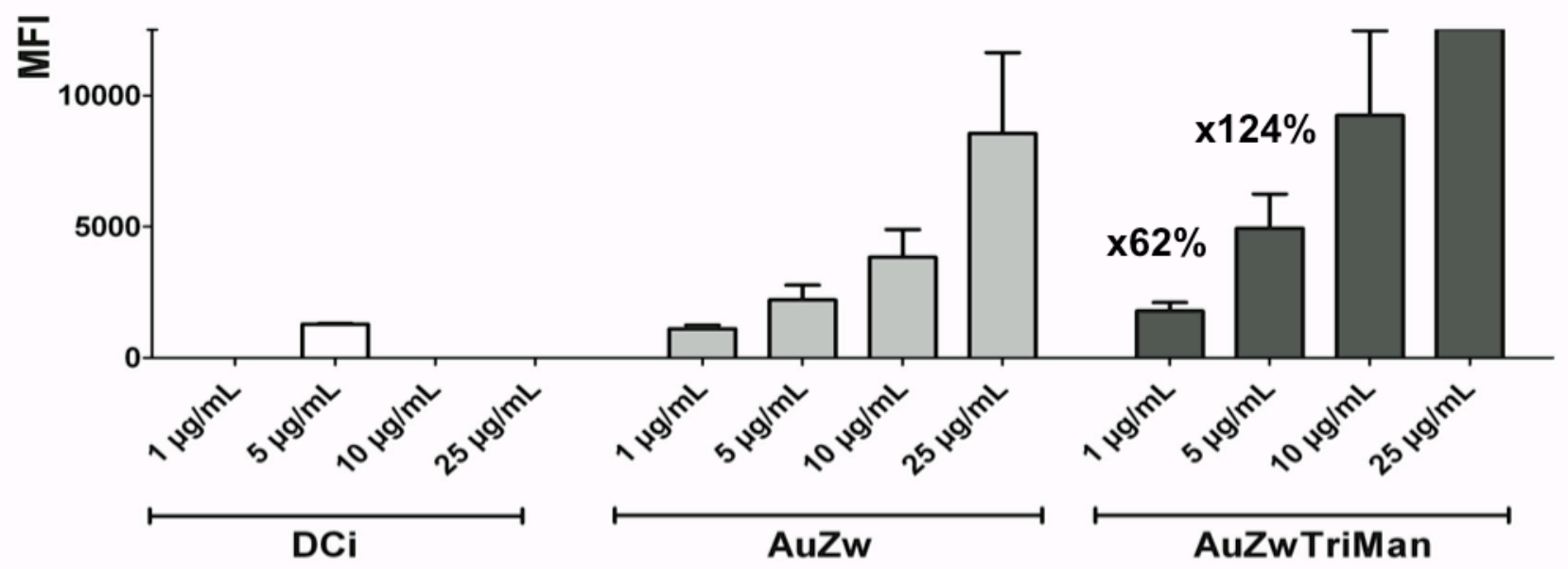

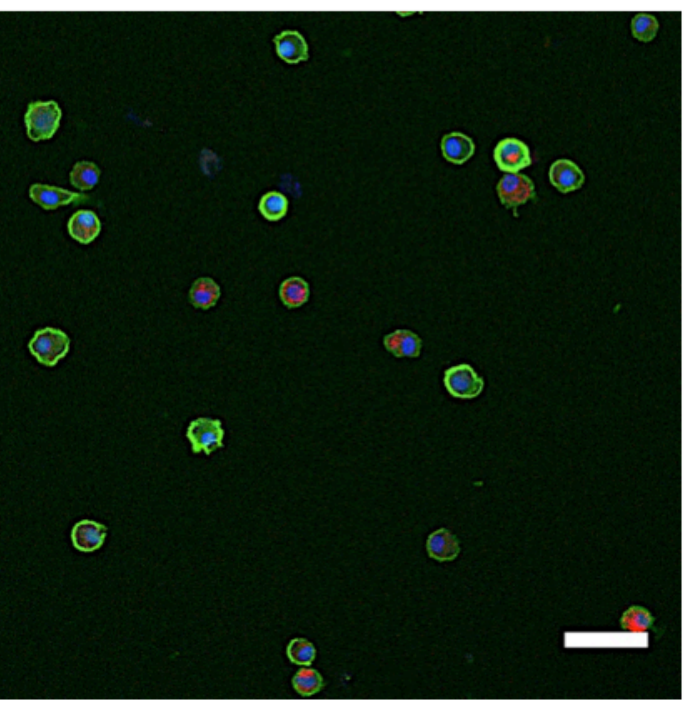

\section{AuZw-5}

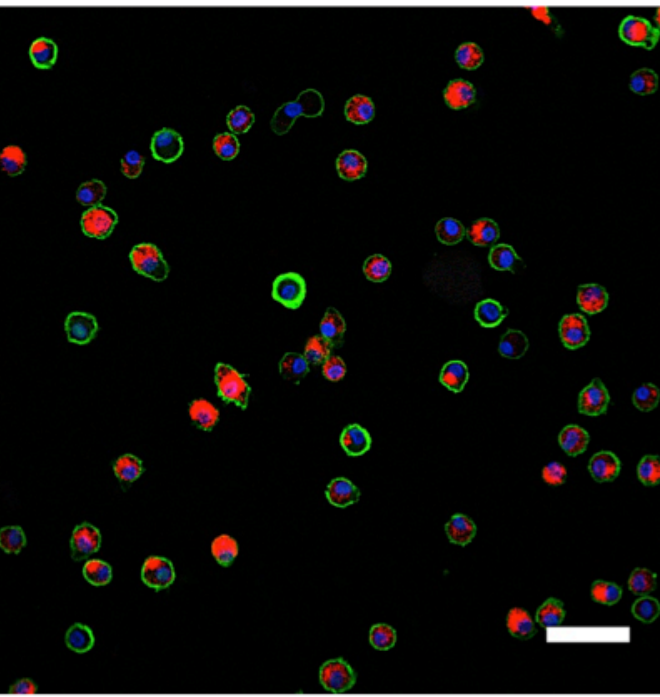

AuZwTriMan-5
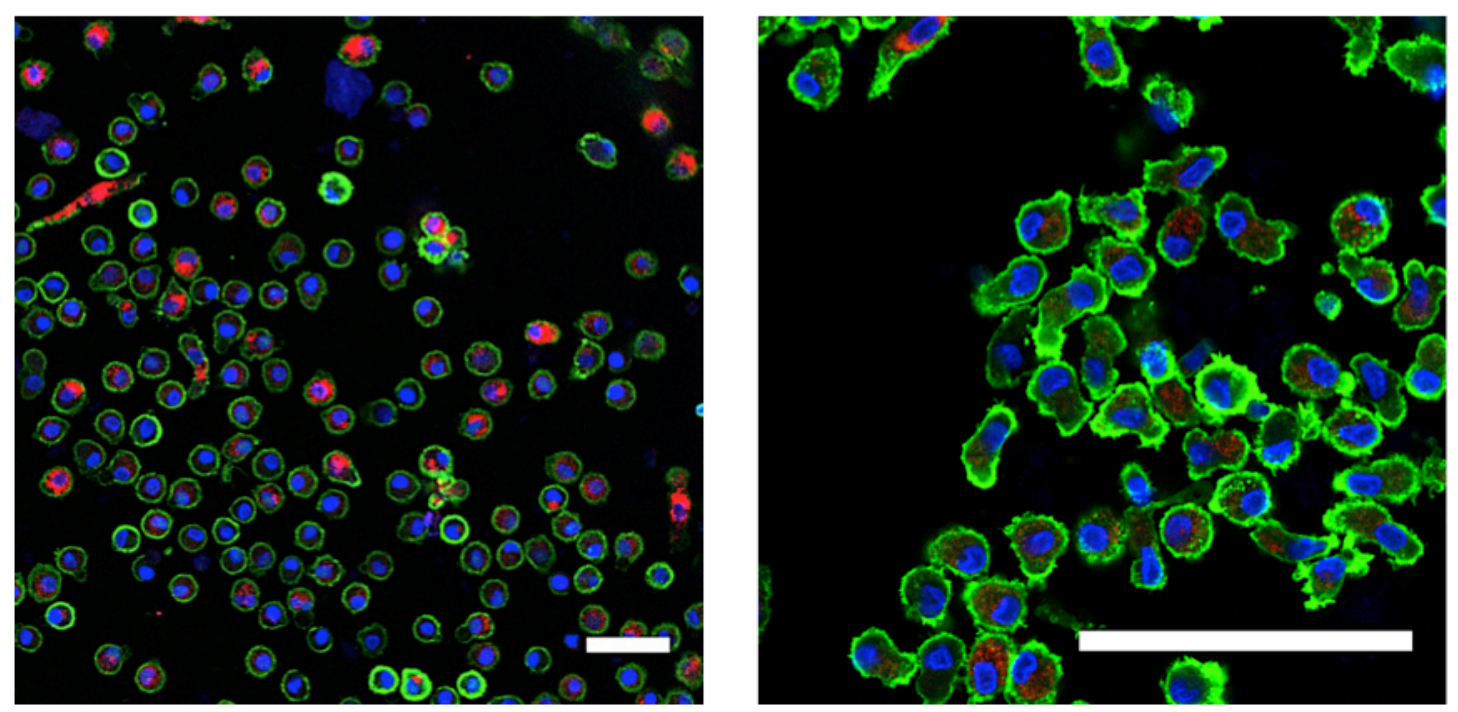

AuZw-10
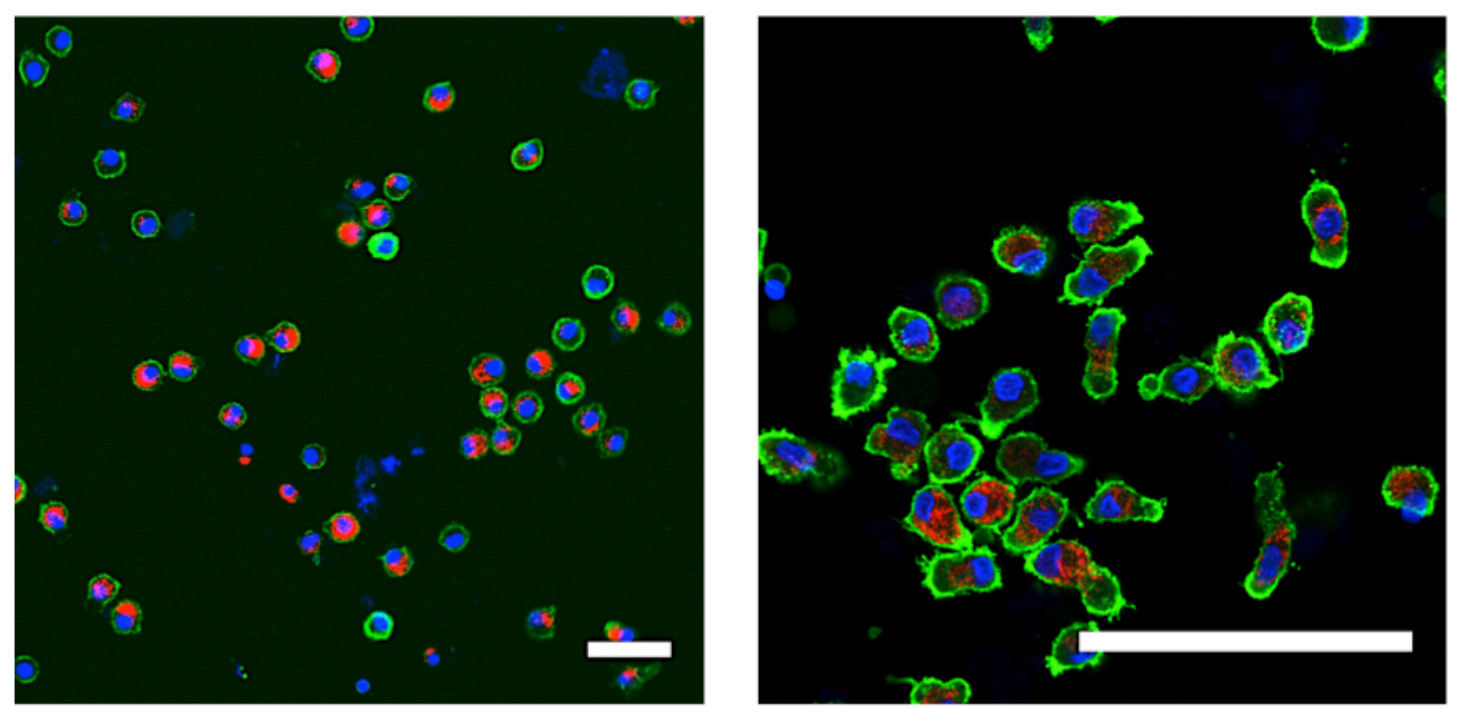

Acs Paragon Plus EnvironmentuZwTriMan-10 


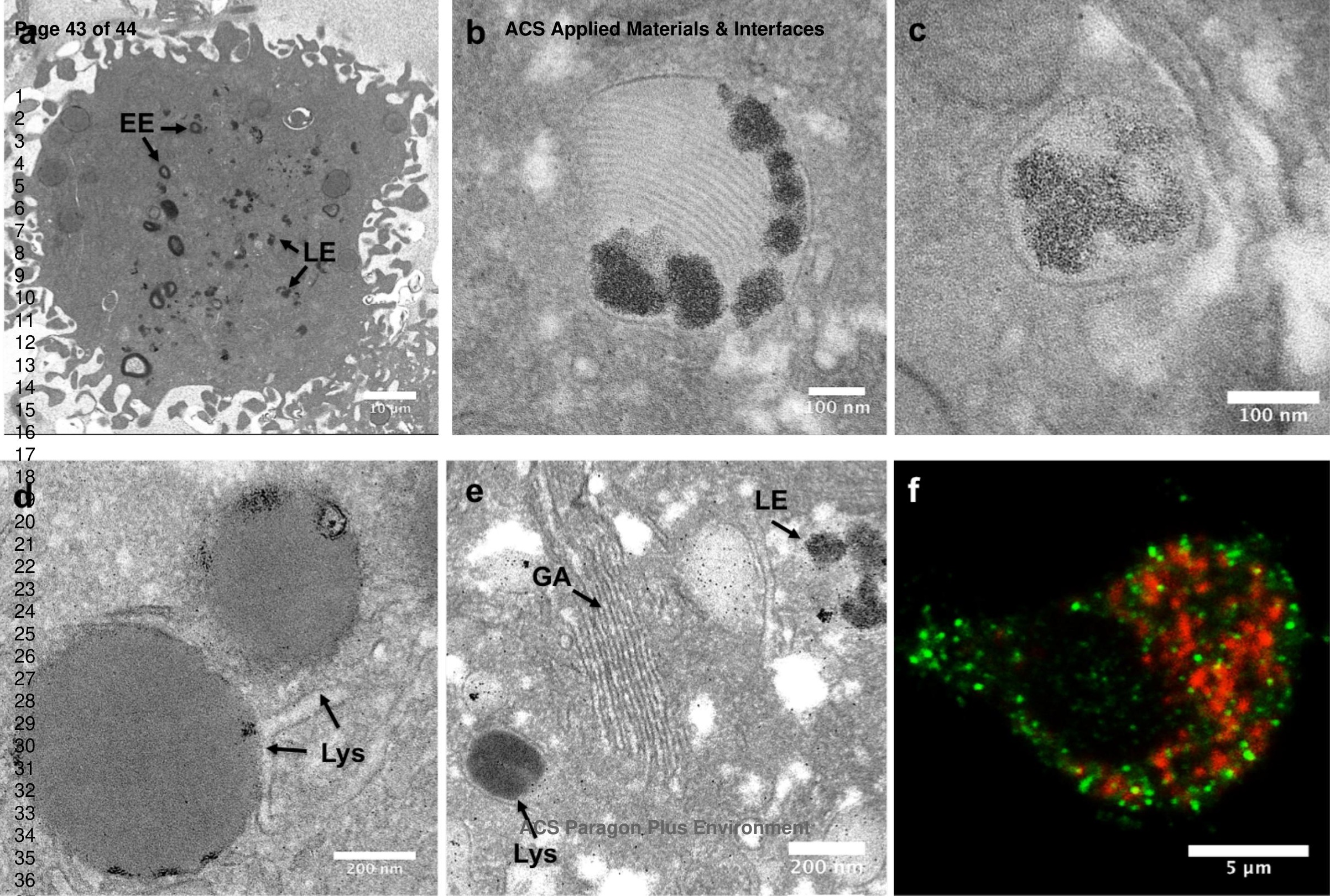


ACS Applied Materials \& Interfaces

$\square$ without inhibitor

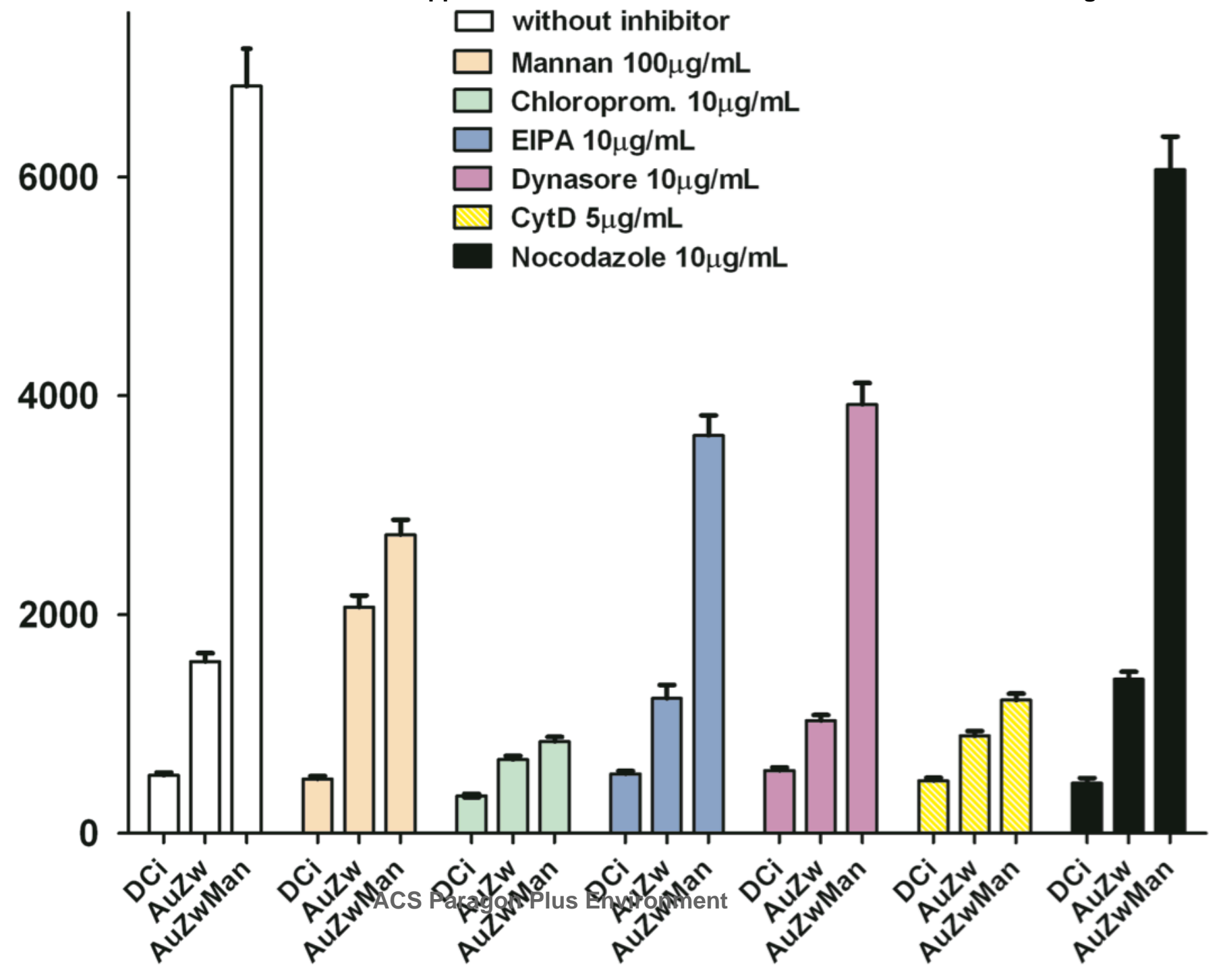

